Supporting Information for the article

\title{
Understanding the F1s NEXAFS Dichroism in Fluorinated Organic Semiconductors
}

M. Klues ${ }^{\text {a) }}$, P. Jerabek ${ }^{\text {b) }}$, T. Breuer ${ }^{\text {a) }}$, M. Oehzeltc), K. Hermann ${ }^{\text {d) }}$, R. Berger $^{\mathrm{b})^{*}}$, and G. Witte ${ }^{\mathrm{a})^{*}}$

a) Fachbereich Physik, Philipps-Universität Marburg, 35032 Marburg, Germany

b) Fachbereich Chemie, Philipps-Universität Marburg, 35032 Marburg, Germany

c) Helmholtz Zentrum für Materialien und Energie GmbH, Hahn-Meitner-Platz

1, 14109 Berlin, Germany

d) Department of Inorganic Chemistry, Fritz-Haber-Institut der Max-PlanckGesellschaft, 14195 Berlin, Germany

*E-Mail: gregor.witte@physik.uni-marburg.de and robert.berger@chemie.unimarburg.de

\section{Contents}

S1 Experimental results $\quad$ S3

S1.1 C1s background subtraction . . . . . . . . . . . . S3

S1.2 F1s NEXAFS spectra of PFP and $\mathrm{K}_{2} \mathrm{TiF}_{6} \ldots \ldots$. . . . . . . S4

S1.3 Perfluoronaphthalene XRD . . . . . . . . . . . S5

S1.4 Perfluoropentacene XPS . . . . . . . . . . . . S6

$\begin{array}{ll}\text { S2 } \text { Theoretical results } & \text { S7 }\end{array}$

S2.1 Perfluoropentacene . . . . . . . . . . . . . S S7

S2.1.1 Partial F1s NEXAFS spectra of Perfluoropentacene . . S7

S2.1.2 Molecular orbitals . . . . . . . . . . . . . S7

S2.1.3 Calculated excitation energies, electric transition dipole moments and oscillator strengths . . . . . . . . S18

S2.1.4 Overlap integrals between ground state and excited states

Slater determinants . . . . . . . . . . . . S20

S2.1.5 HMO Calculations . . . . . . . . . . . . . S22 
S2.1.6 Generalized MO-diagrams of $\pi$ interactions in PFP and the allyl molecule . . . . . . . . . . . . . . . . . . S30

S2.2 Pentacene . . . . . . . . . . . . . . . . . . S S33

S2.2.1 HMO Calculations . . . . . . . . . . . . . . . . S33

S2.3 Perfluoronaphthalene . . . . . . . . . . . . . . . . . . . S37

S2.3.1 Molecular orbitals . . . . . . . . . . . . . . . S37

S2.3.2 Calculated excitations, transition dipole moments and oscillator strengths . . . . . . . . . . . . . S45

S2.3.3 Overlap integrals between ground state and excited states Slater determinants . . . . . . . . . . . . S S46

S3 References 


\section{S1 Experimental results}

\section{S1.1 C1s background subtraction}

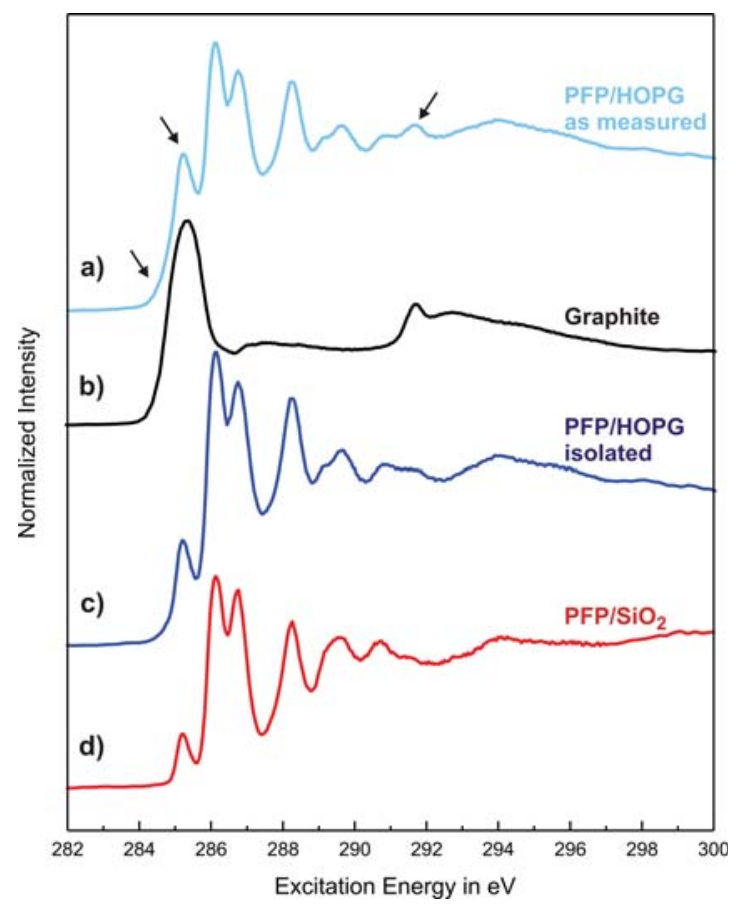

Figure S1: Flow chart of data processing to subtract the C1s NEXAFS signal of the graphite substrate: a) initial NEXAFS spectrum for a 10nm PFP film on HOPG and b) a bare graphite surface. The pure PFP spectrum in c) obtained after weighted subtraction of b) from a) shows the same spectral signature as d) a PFP film grown on $\mathrm{SiO}_{2}$.

In contrast to $\mathrm{SiO}_{2}$ substrates the graphite substrate also causes a distinct C1s signal in the NEXAFS measurements which is observed since the PFP films do not cover the HOPG surface completely. This signal also experiences a strong dichroism and corroborates the reliable analysis of the dichroism of the molecular adlayer. Therefore, a weighted subtraction of the substrate signal is required to remove residual contributions from the substrate as shown in Fig. S1. For this purpose the characteristic $\sigma^{*}$-resonance of graphite at 292 $\mathrm{eV}$ is used to determine the corresponding weighting factor. As shown in Fig. 
S1, this allows reliably isolating the signature of the adlayer and subsequently analyzing the corresponding dichroism. Further details on this approach are provided in [6] and [10].

\section{S1.2 F1s NEXAFS spectra of PFP and $\mathrm{K}_{2} \mathrm{TiF}_{6}$}

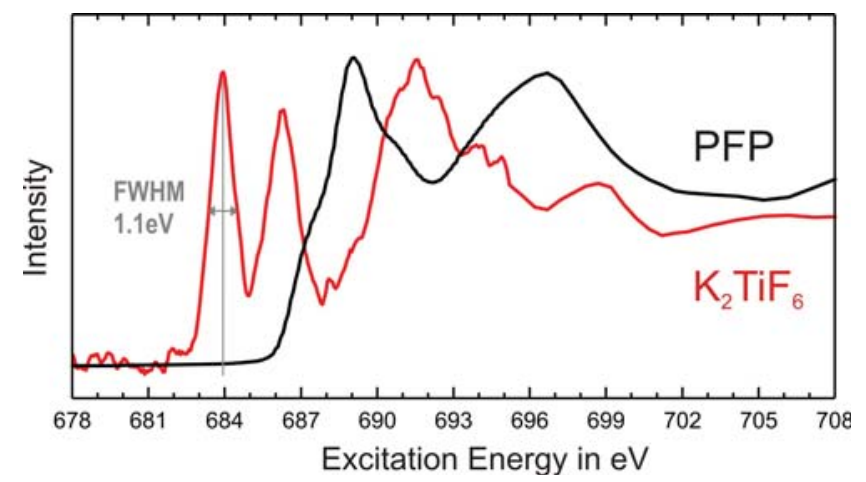

Figure S2: F1s NEXAFS spectra of PFP and $\mathrm{K}_{2} \mathrm{TiF}_{6}$.

$\mathrm{K}_{2} \mathrm{TiF}_{6}$ shows two leading, sharp d-type resonances. For these peaks a FWHM of $1.1 \mathrm{eV}$ is observed, meaning that the predicted FWHM of $1.88 \mathrm{eV}$ for $\sigma$ resonances is not given by resolution limitations of the beamline. Furthermore the literature known position of these peaks was used for energy calibration $[11][12]$. 


\section{S1.3 Perfluoronaphthalene XRD}

Perfluoronaphthalene thin films were prepared by freezing molecules from gas phase onto a cooled $\mathrm{SiO}_{2}$ substrate. The PFN thin film is unstable under ambient conditions and desorbs in about a hour depending on initial thickness. Therefore XRD-measurements needed to be done quiet fast, explaining the low signal to noise ratio. Never the less several crystallographic orientations can be observed as depicted in Fig S3. Below the miller indices the relative signal strength compared to the most intense reflex (11-3) is given, which can be calculated from the literature known crystal structure of PFN [20]. As the (002)-plane has a more than fifty times smaller diffraction strength than the (11-3)-, (012)- or (11-2)-plane but shows nearly the same intensity in Fig.S3 it can be concluded, that the thin film is mainly oriented in (002) direction.

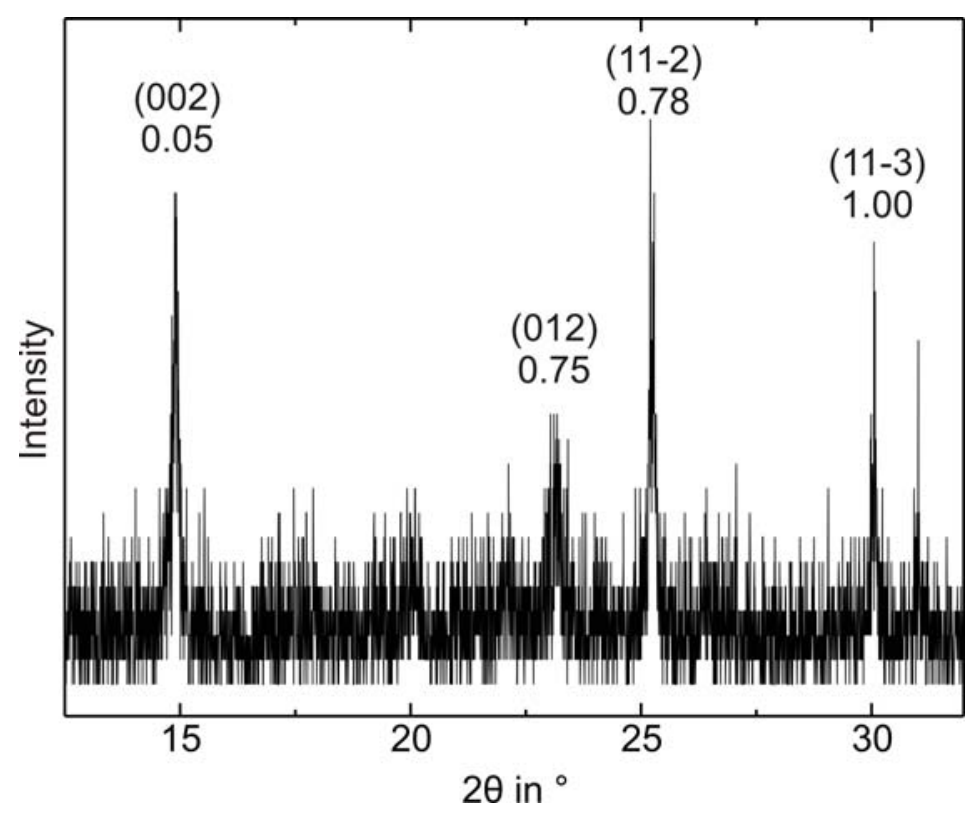

Figure S3: Diffractogram of Perfluoronaphthalene thin film. Reflexes are labeled corresponding to [20]. 


\section{S1.4 Perfluoropentacene XPS}

In Fig.S4 the energy difference between F1s and C1s-signals of perfluoropentacene measured by x-ray photoemission can be seen. Using the calculated ionisation potentials for the various excitation centres similar differences for the StoBe-results can be obtained, yielding values of $400.67 \mathrm{eV}$ and $399.40 \mathrm{eV}$. Both numbers are smaller then the experimental differences by around $1 \mathrm{eV}$. Since the calculated ionisation potentials for the case of carbon are accurate within $0.3 \mathrm{eV}$, the deviation must be due to errors in the calculations for F1sexcitation centres. Averaging leads to a shift between calculated and experimental energy differences of about $1.17 \mathrm{eV}$. This justifies the empirical shift of $1.2 \mathrm{eV}$, which was necessary to compare the calculated and experimental NEXAFS-spectra.

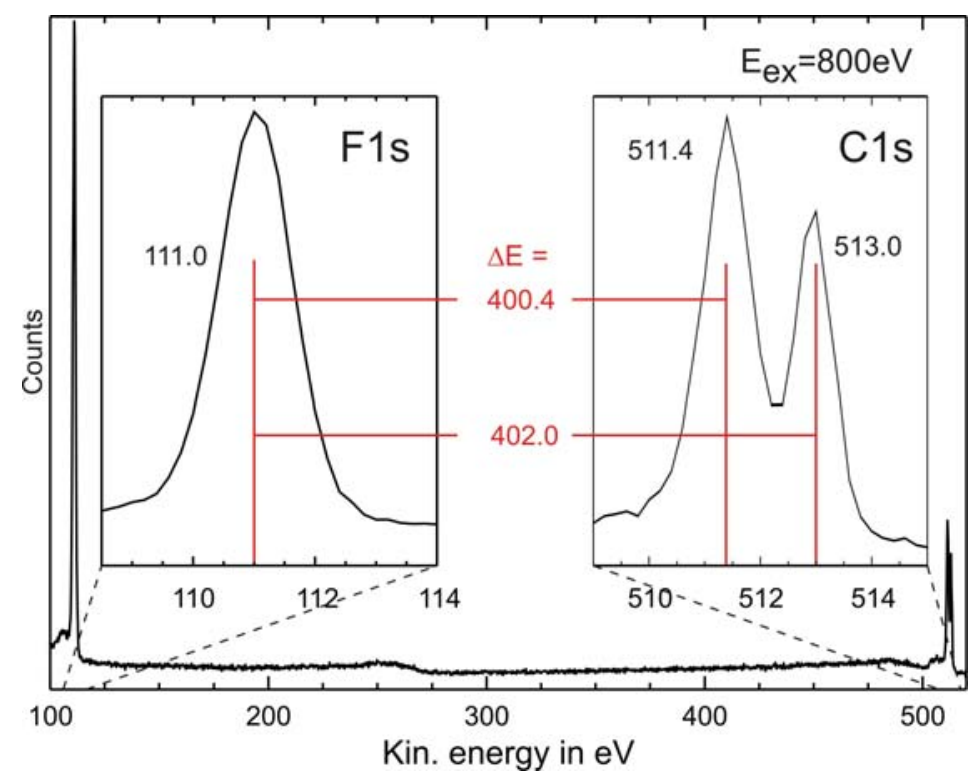

Figure S4: X-ray photoemission spectrum of Perfluoropentacene. Insets show magnifications of the F1s- and C1s-region. In red the energy differences of these peaks are given. 


\section{S2 Theoretical results}

\section{S2.1 Perfluoropentacene}

\section{S2.1.1 Partial F1s NEXAFS spectra of Perfluoropentacene}

The StoBe-code calculates partial NEXAFS spectra for all symmetry inequivalent excitation centres. To compare the calculation with experimental data a total spectrum is calculated by summing up the partial ones. Therefore the multiplicity needs to be considered. All excitation centres can be found four times in a perfluoropentacene molecule except the F1-centre, which is represented only two times. Therefore the F1-spectrum has only half of the intensity of the spectra from the other centres within the full spectrum.

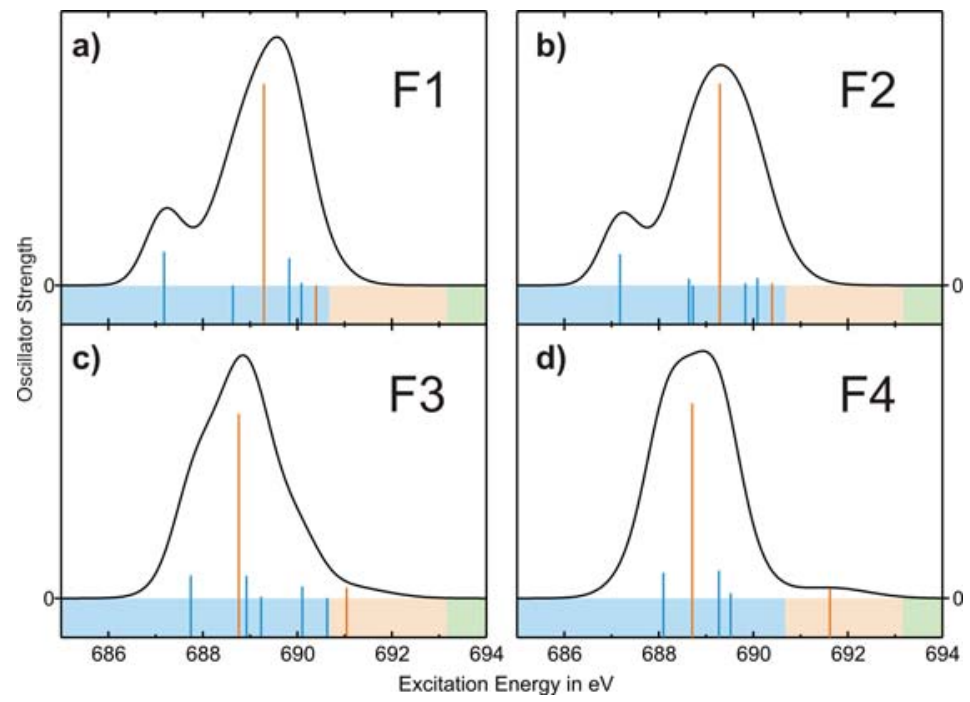

Figure S5: Partial F1s NEXAFS spectra for all symmetry inequivalent excitation centres of perfluoropentacene. $\pi$-resonances are depicted as blue bars, while $\sigma$-resonances are given in orange.

\section{S2.1.2 Molecular orbitals}

The figures in this subsection show select Kohn-Sham molecular orbitals as obtained for perfluoropentacene with the def2-SVP basis set and the PBE density functional as implemented in the program package Turbomole 6.6 [23], [24], utilizing the RI approximation [23]-[25]. The Kohn-Sham molecular orbitals 
in Figs. S6-S8 were obtained in Kohn-Sham DFT calculations for the electronic ground state. The molecular orbitals shown in Figs. S9-S12 are the results from individual self-consistent field (SCF) calculations that essentially describe single electron excitations from localized F1s core orbitals (centered at F1, F2, F3 or F4 which are indicated by the green color, whereas other fluorine atoms are shown in blue color; see also main text for the numbering convention) into different unoccupied molecular orbitals. For each of these SCF calculations only the obtained energetically high-lying molecular orbital, into which the electron is promoted, is shown. This orbital we loosely refer to as a singly occupied molecular orbital (SOMO), as if it were obtained from a restricted open-shell SCF calculation. Note that the actual calculations for excited states were performed in an unrestricted Kohn-Sham framework. The initial Kramers degeneracy of the ground state Kohn-Sham orbitals is thereby lifted such that essentially all occupied molecular orbitals become singly occupied spin-orbitals (different orbitals for different spin, DODS) instead of doubly occupied or singly occupied spatial orbitals. Neither the corresponding relaxed core holes obtained in each of these unrestricted SCF procedures nor the corresponding orbitals with opposite spin are explicitly shown.

The molecular orbitals shown in the first set below are linear combinations of the fluorine core orbitals (Fig. S6). To the second set of molecular orbitals belong energetically low-lying $\pi^{*}$ type Kohn-Sham molecular orbitals (Fig. S7), with LUMO referring to the energetically lowest unoccupied molecular orbital in the electronic groundstate and $\mathrm{LUMO}+1$ referring to the next higher energy molecular orbital. The third set of molecular orbitals contains $\sigma^{*}$ type Kohnmolecular orbitals (Fig. S8). The orbitals of these first three sets were obtained in Kohn-Sham DFT calculations for the electronic ground state, whereas the forth, fifth, sixth and seventh set (Figs. S9-S12) feature SOMOs from SCF calculations for core-hole excited states. The molecular orbitals are displayed as contour surfaces for a given isovalue $\left(0.05 a_{0}^{-3 / 2}\right)$ of the corresponding oneelectron wavefunction. The different colors (blue $(+1)$ and red $(-1)$ ) indicate the sign of the isovalue. 


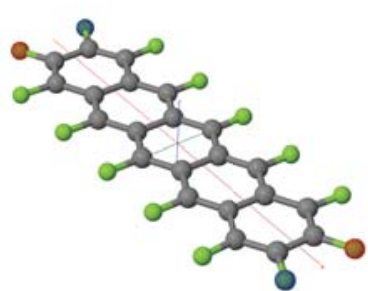

MO 1

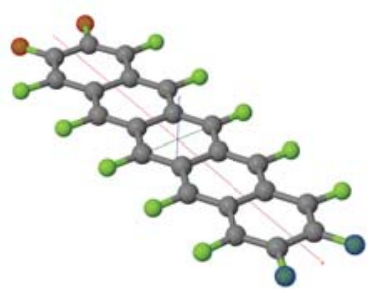

MO 3

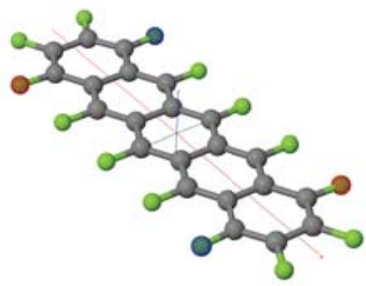

MO 5

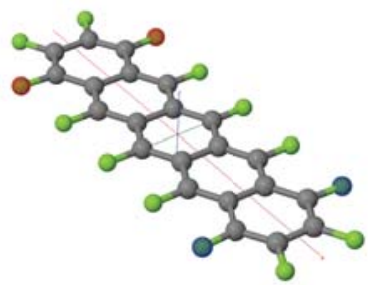

MO 7

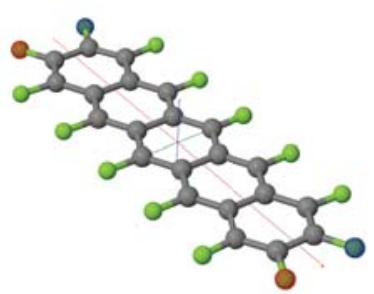

MO 2

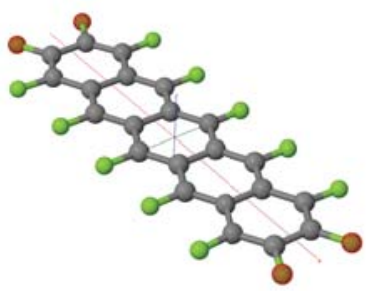

$\mathrm{MO} 4$

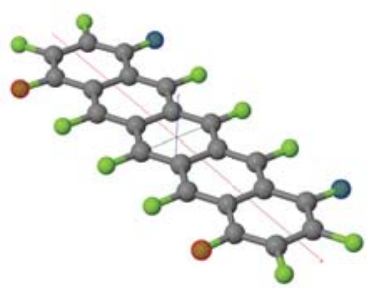

MO 6

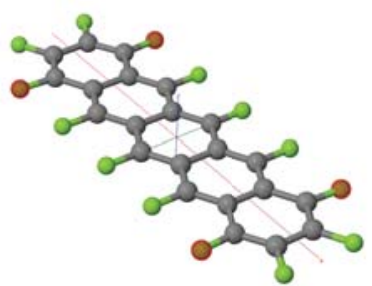

MO 8

Figure S6: PFP fluorine core electron MOs. 


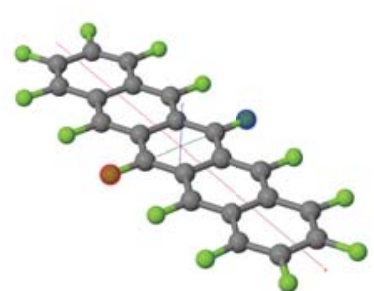

MO 9

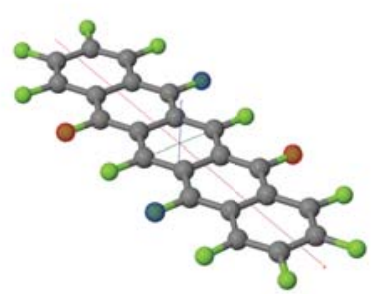

MO 11

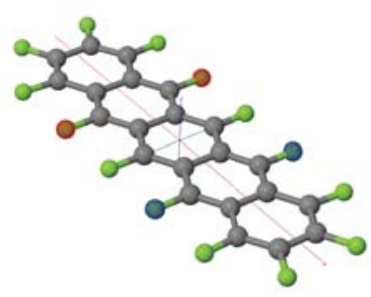

MO 13

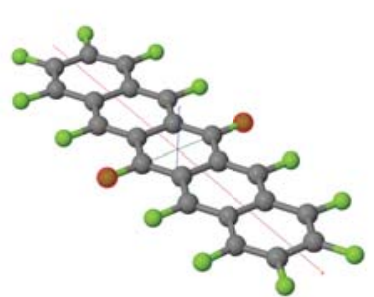

MO 10

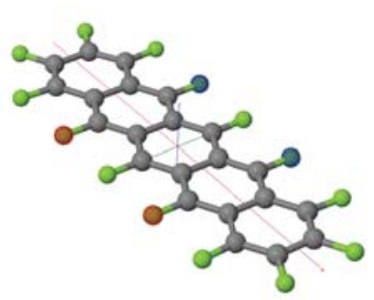

MO 12

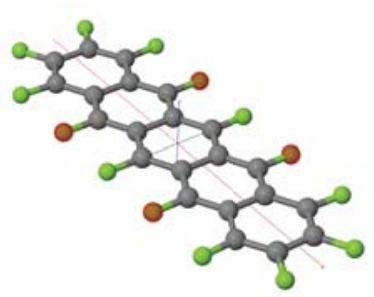

MO 14

Figure S6 (Cont.): PFP fluorine core electron MOs. 


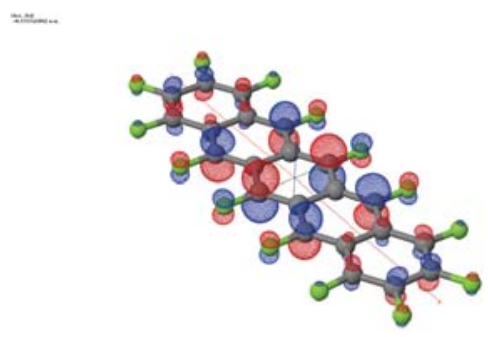

LUMO

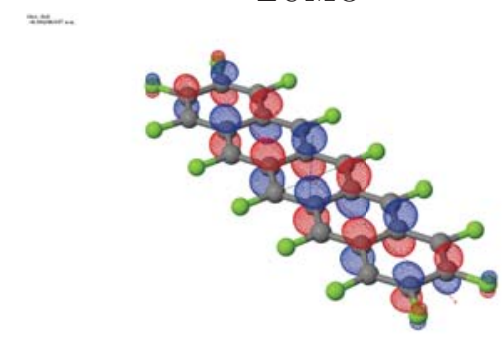

$\mathrm{LUMO}+2$

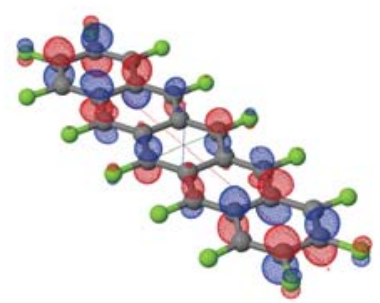

$\mathrm{LUMO}+4$

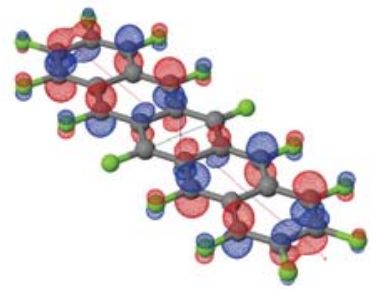

$\mathrm{LUMO}+1$

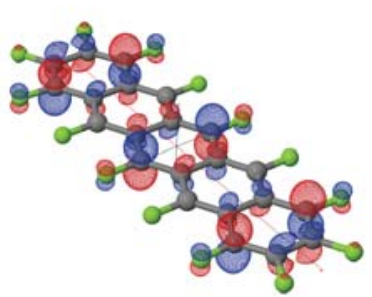

$\mathrm{LUMO}+3$

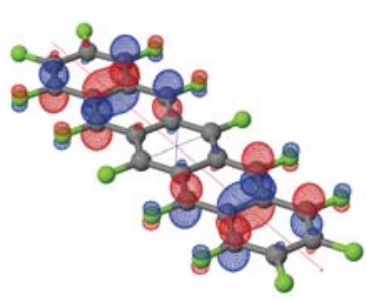

$\mathrm{LUMO}+7$

Figure S7: PFP $\pi^{*}$ type unoccupied MOs. 


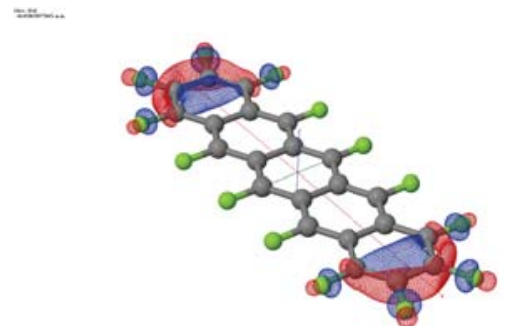

$\mathrm{LUMO}+5$

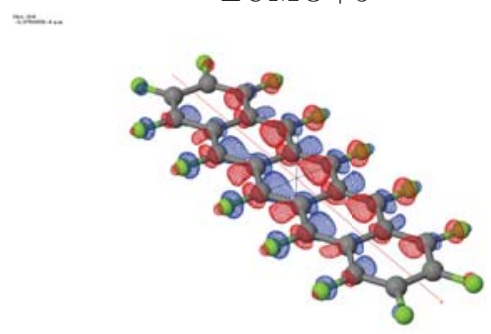

$\mathrm{LUMO}+9$

$=$

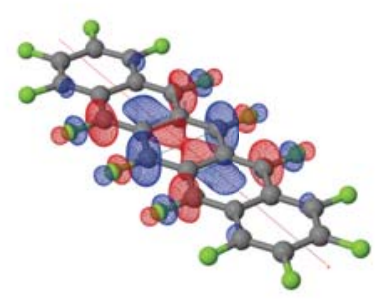

$$
\text { LUMO+12 }
$$

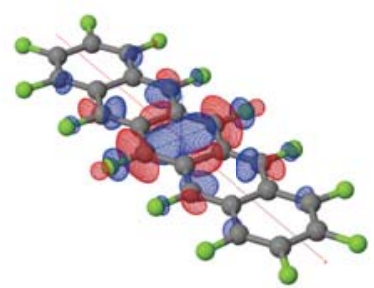

$\mathrm{LUMO}+14$

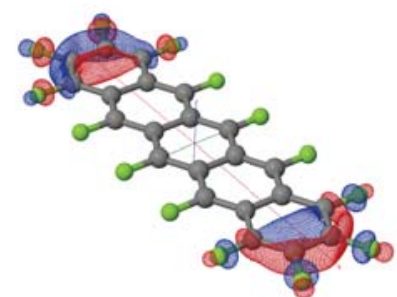

$\mathrm{LUMO}+6$

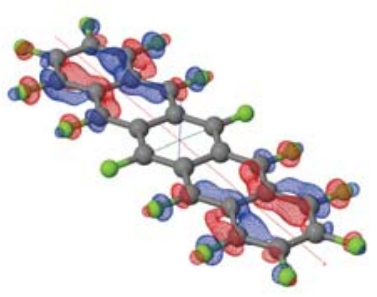

$\mathrm{LUMO}+11$

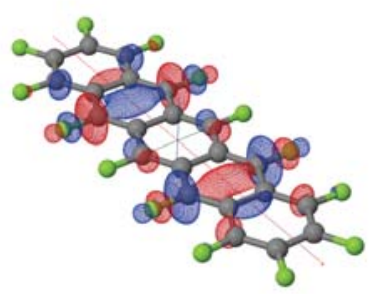

LUMO+13

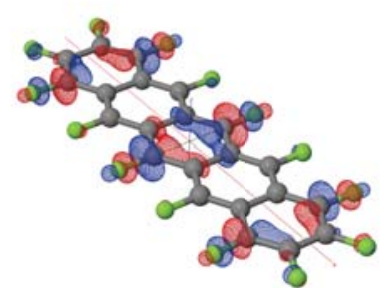

$\mathrm{LUMO}+15$

Figure S8: PFP $\sigma^{*}$ type unoccupied MOs. 


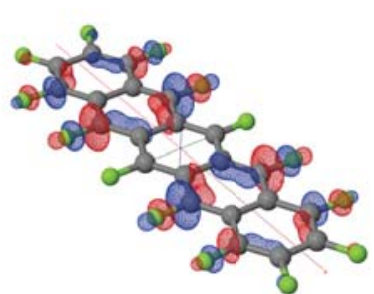

$\mathrm{LUMO}+17$

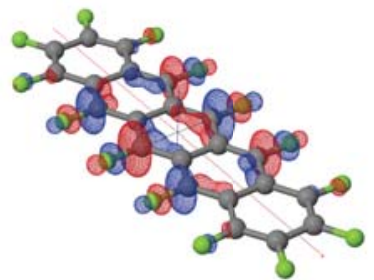

LUMO +18

Figure S8 (Cont.): PFP $\sigma^{*}$ type unoccupied MOs. 


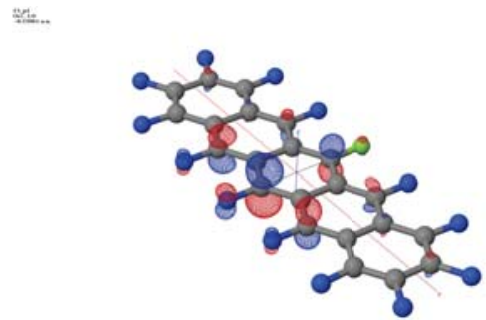

$\pi_{1}$
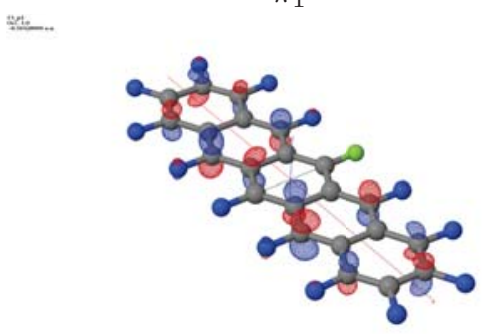

$\pi_{3}$

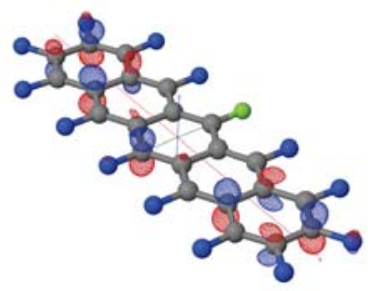

$\pi_{5}$
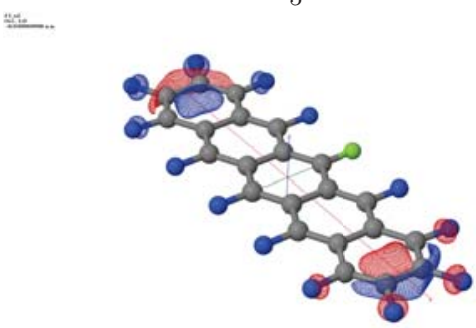

$\sigma_{2}$

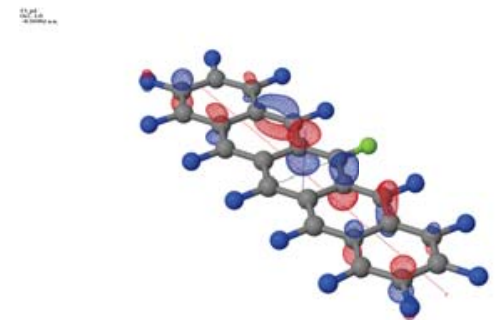

$\pi_{2}$

ne.

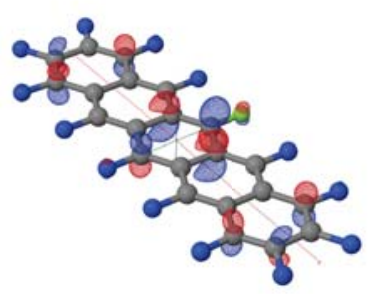

$\pi_{4}$

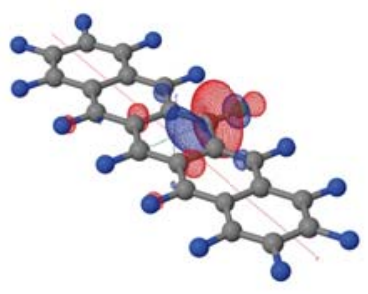

$\sigma_{1}$

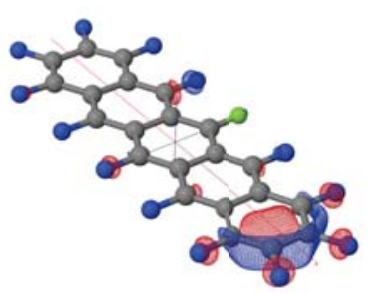

$\sigma_{3}$

Figure S9: SOMOs of PFP (resulting from F1 core electron excitations) for the transitions into the lowest-lying MOs. 
和

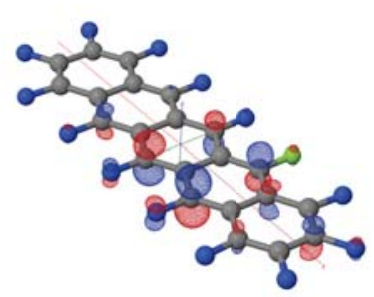

$\pi_{1}$

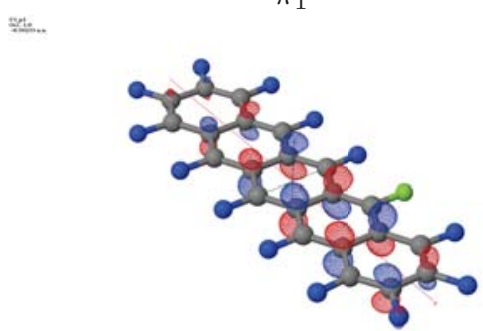

$\pi_{3}$

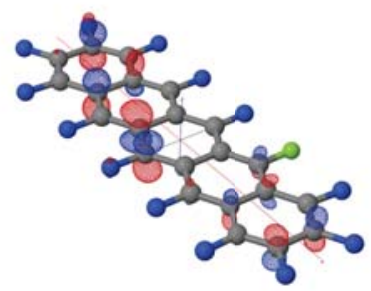

$\pi_{5}$

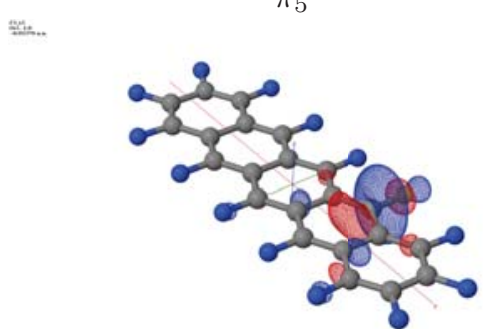

$\sigma_{1}$
雌.

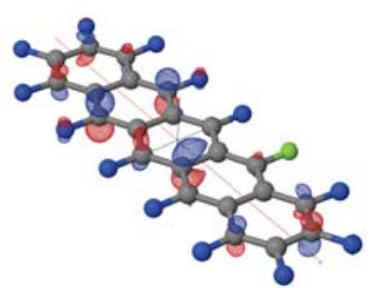

$\pi_{2}$

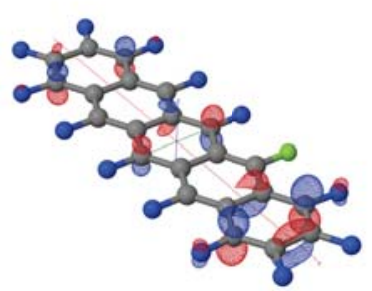

$\pi_{4}$

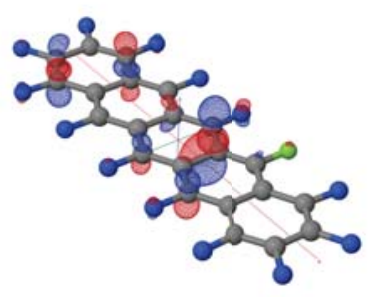

$\pi_{6}$

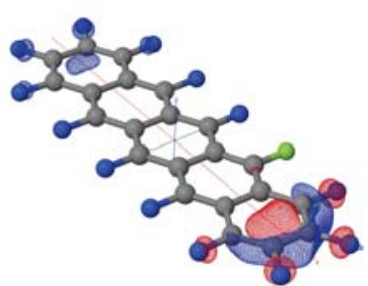

$\sigma_{2}$

Figure S10: SOMOs of PFP (resulting from F2 core electron excitations) for the transitions into the lowest-lying MOs. 

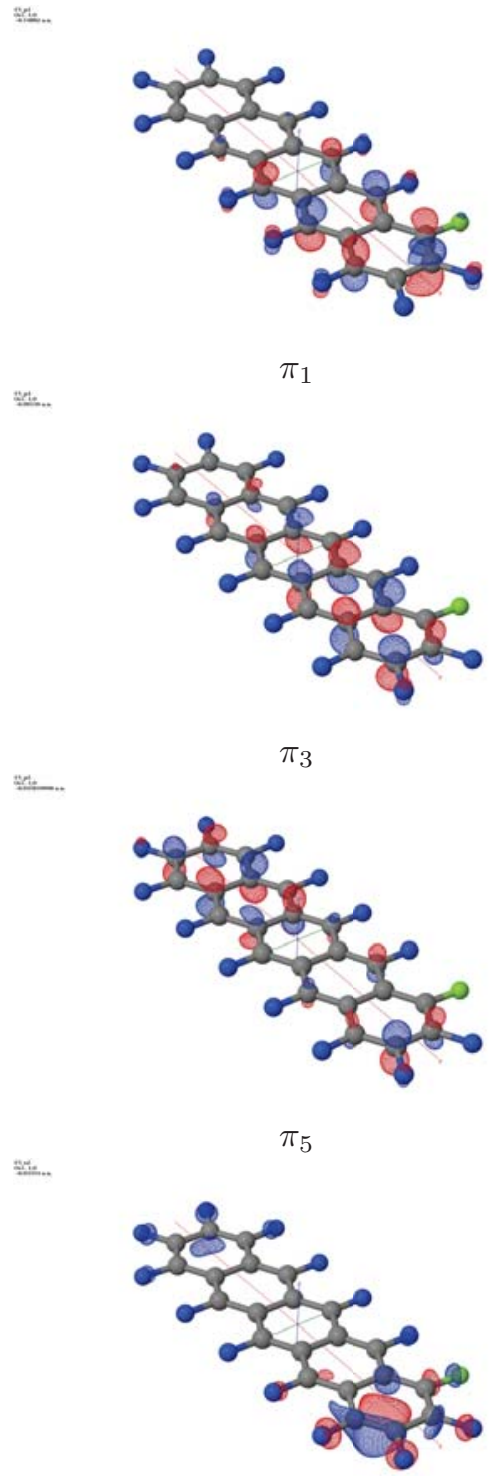

$\sigma_{2}$

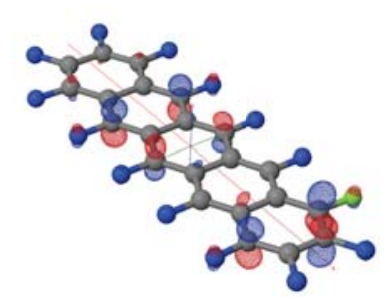

$\pi_{2}$

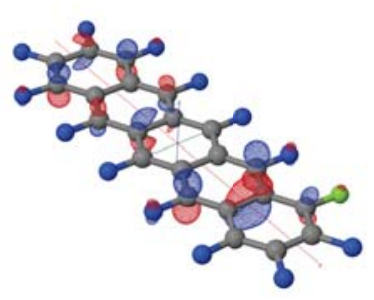

$\pi_{4}$

뽀.

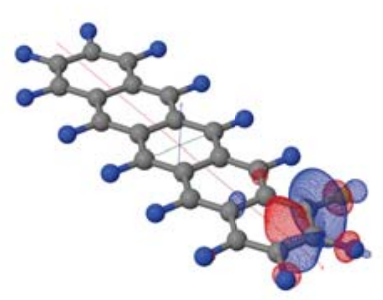

$\sigma_{1}$

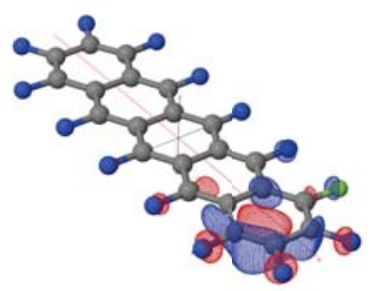

$\sigma_{3}$

Figure S11: SOMOs of PFP (resulting from F3 core electron excitations) for the transitions into the lowest-lying MOs. 


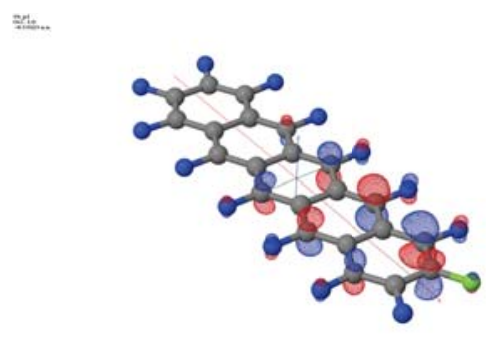

$\pi_{1}$

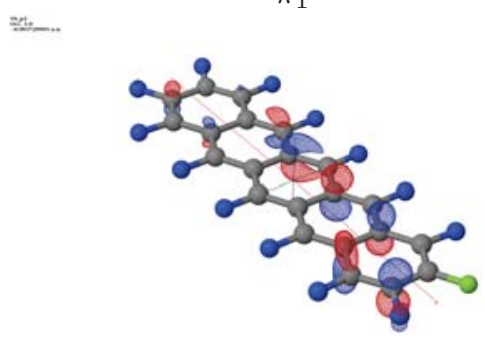

$\pi_{3}$

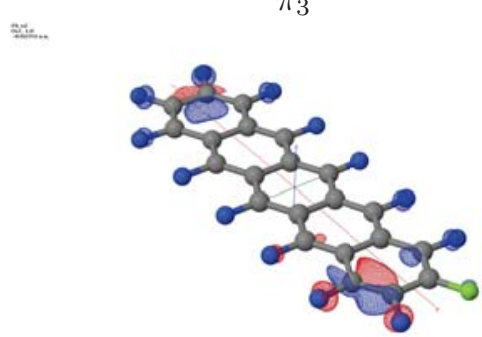

$\sigma_{2}$ yㅡ.

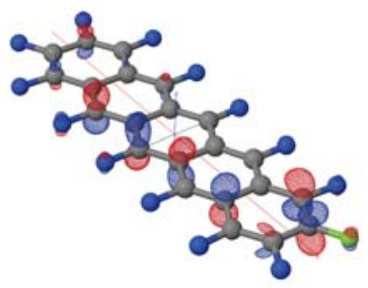

$\pi_{2}$

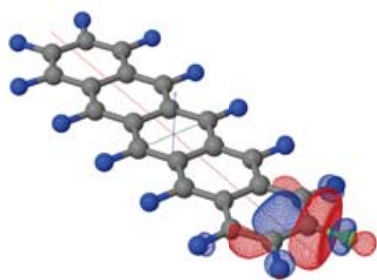

$\sigma_{1}$

Figure S12: SOMOs of PFP (resulting from F4 core electron excitations) for the transitions into the lowest-lying MOs. 
S2.1.3 Calculated excitation energies, electric transition dipole moments and oscillator strengths

Table S1: PFP F1 core electron excitation energies, oscillator strengths and transition dipole moment components for the transition into the lowest lying unoccupied MOs. Method: $\triangle \mathrm{SCF}$, revPBE/StoBe basis set (TZ quality).

\begin{tabular}{ccrrrr}
\hline Exc. & $\varepsilon / \mathbf{e V}$ & Osc. Str. & TDM $_{\mathbf{x}}$ & TDM $_{\mathbf{y}}$ & \multicolumn{1}{c}{$\mathbf{T D M}_{\mathbf{z}}$} \\
\hline$\pi_{1}$ & 685.580 & 0.001797 & 0.000000 & 0.000000 & -0.010354 \\
$\pi_{2}$ & 687.036 & 0.000000 & 0.000000 & 0.000000 & 0.000000 \\
$\pi_{3}$ & 687.121 & 0.000000 & 0.000000 & 0.000000 & 0.000019 \\
$\pi_{4}$ & 688.228 & 0.001467 & 0.000000 & 0.000000 & 0.009333 \\
$\pi_{5}$ & 688.487 & 0.000135 & 0.000000 & 0.000000 & 0.002812 \\
$\sigma_{1}$ & 687.692 & 0.010833 & 0.000000 & -0.025357 & 0.000000 \\
$\sigma_{2}$ & 688.795 & 0.000000 & -0.000273 & -0.000005 & 0.000000 \\
$\sigma_{3}$ & 689.236 & 0.000304 & -0.000778 & 0.004140 & 0.000000 \\
\hline
\end{tabular}

Table S2: PFP F2 core electron excitation energies, oscillator strengths and transition dipole moment components for the transition into the lowest lying unoccupied MOs. Method: $\triangle \mathrm{SCF}$, revPBE/StoBe basis set (TZ quality).

\begin{tabular}{ccrrrr}
\hline Exc. & $\varepsilon / \mathbf{e V}$ & Osc. Str. & TDM $_{\mathbf{x}}$ & \multicolumn{1}{c}{ TDM $_{\mathbf{y}}$} & \multicolumn{1}{c}{ TDM $_{\mathbf{z}}$} \\
\hline$\pi_{1}$ & 685.580 & 0.001680 & 0.000000 & 0.000000 & 0.010006 \\
$\pi_{2}$ & 687.036 & 0.000353 & 0.000000 & 0.000000 & 0.004578 \\
$\pi_{3}$ & 687.121 & 0.000000 & 0.000000 & 0.000000 & 0.000387 \\
$\pi_{4}$ & 688.228 & 0.000101 & 0.000000 & 0.000000 & -0.002426 \\
$\pi_{5}$ & 688.487 & 0.000388 & 0.000000 & 0.000000 & -0.004780 \\
$\pi_{6}$ & 688.796 & 0.000557 & 0.000000 & 0.000000 & 0.005733 \\
$\sigma_{1}$ & 687.692 & 0.010850 & -0.000258 & -0.025379 & 0.000000 \\
$\sigma_{2}$ & 688.795 & 0.000118 & 0.000407 & 0.002547 & 0.000000 \\
\hline
\end{tabular}


Table S3: PFP F3 core electron excitation energies, oscillator strengths and transition dipole moment components for the transition into the lowest lying unoccupied MOs. Method: $\triangle \mathrm{SCF}$, revPBE/StoBe basis set (TZ quality).

\begin{tabular}{ccrrrr}
\hline Exc. & $\varepsilon / \mathbf{e V}$ & Osc. Str. & \multicolumn{1}{c}{ TDM $_{\mathbf{x}}$} & \multicolumn{1}{c}{ TDM $_{\mathbf{y}}$} & \multicolumn{1}{c}{$\mathbf{T D M}_{\mathbf{z}}$} \\
\hline$\pi_{1}$ & 686.145 & 0.001227 & 0.000000 & 0.000000 & -0.008573 \\
$\pi_{2}$ & 687.323 & 0.001212 & 0.000000 & 0.000000 & 0.008502 \\
$\pi_{3}$ & 687.632 & 0.000084 & 0.000000 & 0.000000 & -0.002271 \\
$\pi_{4}$ & 688.506 & 0.000641 & 0.000000 & 0.000000 & -0.006194 \\
$\pi_{5}$ & 689.034 & 0.000017 & 0.000000 & 0.000000 & -0.000957 \\
$\sigma_{1}$ & 687.162 & 0.009916 & 0.000683 & 0.024260 & 0.000000 \\
$\sigma_{2}$ & 689.442 & 0.000574 & -0.003415 & -0.004720 & 0.000000 \\
$\sigma_{3}$ & 689.554 & 0.000557 & 0.004534 & 0.003536 & 0.000000 \\
\hline
\end{tabular}

Table S4: PFP F4 core electron excitation energies, oscillator strengths and transition dipole moment components for the transition into the lowest lying unoccupied MOs. Method: $\triangle \mathrm{SCF}$, revPBE/StoBe basis set (TZ quality).

\begin{tabular}{ccrccr}
\hline Exc. & $\varepsilon / \mathbf{e V}$ & Osc. Str. & TDM $_{\mathbf{x}}$ & $\mathbf{T D M}_{\mathbf{y}}$ & \multicolumn{1}{c}{$\mathbf{T D M}_{\mathbf{z}}$} \\
\hline$\pi_{1}$ & 686.497 & 0.001379 & 0.000000 & 0.000000 & 0.009044 \\
$\pi_{2}$ & 687.671 & 0.001499 & 0.000000 & 0.000000 & -0.009420 \\
$\pi_{3}$ & 687.921 & 0.000270 & 0.000000 & 0.000000 & 0.003971 \\
$\sigma_{1}$ & 687.107 & 0.010487 & 0.021964 & 0.011853 & 0.000000 \\
$\sigma_{2}$ & 690.020 & 0.000541 & 0.004251 & -0.003686 & 0.000000 \\
$\sigma_{3}$ & 690.169 & 0.000693 & 0.003854 & -0.005155 & 0.000000 \\
\hline
\end{tabular}


S2.1.4 Overlap integrals between ground state and excited states Slater determinants

Table S5: Absolute value of the overlap integral between the PFP ground state and F1 core electron excited Slater determinants.

\begin{tabular}{c|ccccccccc}
\hline & GS & $\pi_{1}$ & $\pi_{2}$ & $\pi_{3}$ & $\pi_{4}$ & $\pi_{5}$ & $\sigma_{1}$ & $\sigma_{2}$ & $\sigma_{3}$ \\
\hline GS & 1.000 & 0.000 & 0.000 & 0.001 & 0.000 & 0.000 & 0.002 & 0.000 & 0.000 \\
$\pi_{1}$ & 0.000 & 1.000 & 0.000 & 0.001 & 0.023 & 0.018 & 0.000 & 0.000 & 0.000 \\
$\pi_{2}$ & 0.000 & 0.000 & 1.000 & 0.324 & 0.000 & 0.000 & 0.000 & 0.000 & 0.000 \\
$\pi_{3}$ & 0.000 & 0.001 & 0.324 & 1.000 & 0.001 & 0.001 & 0.000 & 0.000 & 0.000 \\
$\pi_{4}$ & 0.000 & 0.023 & 0.000 & 0.001 & 1.000 & 0.008 & 0.000 & 0.000 & 0.000 \\
$\pi_{5}$ & 0.000 & 0.018 & 0.000 & 0.001 & 0.008 & 1.000 & 0.000 & 0.000 & 0.000 \\
$\sigma_{1}$ & 0.002 & 0.000 & 0.000 & 0.000 & 0.000 & 0.000 & 1.000 & 0.000 & 0.005 \\
$\sigma_{2}$ & 0.000 & 0.000 & 0.000 & 0.000 & 0.000 & 0.000 & 0.000 & 1.000 & 0.543 \\
$\sigma_{3}$ & 0.000 & 0.000 & 0.000 & 0.000 & 0.000 & 0.000 & 0.005 & 0.543 & 1.000 \\
\hline
\end{tabular}

Table S6: Absolute value of the overlap integral between the PFP ground state and F2 core electron excited Slater determinants.

\begin{tabular}{c|ccccccccc}
\hline & GS & $\pi_{1}$ & $\pi_{2}$ & $\pi_{3}$ & $\pi_{4}$ & $\pi_{5}$ & $\pi_{6}$ & $\sigma_{1}$ & $\sigma_{2}$ \\
\hline GS & 1.000 & 0.000 & 0.000 & 0.000 & 0.000 & 0.000 & 0.000 & 0.002 & 0.000 \\
$\pi_{1}$ & 0.000 & 1.000 & 0.097 & 0.004 & 0.041 & 0.015 & 0.002 & 0.000 & 0.000 \\
$\pi_{2}$ & 0.000 & 0.097 & 1.000 & 0.255 & 0.100 & 0.012 & 0.021 & 0.000 & 0.000 \\
$\pi_{3}$ & 0.000 & 0.004 & 0.255 & 1.000 & 0.014 & 0.080 & 0.019 & 0.000 & 0.000 \\
$\pi_{4}$ & 0.000 & 0.041 & 0.100 & 0.014 & 1.000 & 0.184 & 0.352 & 0.000 & 0.000 \\
$\pi_{5}$ & 0.000 & 0.015 & 0.012 & 0.080 & 0.184 & 1.000 & 0.064 & 0.000 & 0.000 \\
$\pi_{6}$ & 0.000 & 0.002 & 0.021 & 0.019 & 0.352 & 0.064 & 1.000 & 0.000 & 0.000 \\
$\sigma_{1}$ & 0.002 & 0.000 & 0.000 & 0.000 & 0.000 & 0.000 & 0.000 & 1.000 & 0.061 \\
$\sigma_{2}$ & 0.000 & 0.000 & 0.000 & 0.000 & 0.000 & 0.000 & 0.000 & 0.061 & 1.000 \\
\hline
\end{tabular}


Table S7: Absolute value of the overlap integral between the PFP ground state and F3 core electron excited Slater determinants.

\begin{tabular}{c|ccccccccc}
\hline & GS & $\pi_{1}$ & $\pi_{2}$ & $\pi_{3}$ & $\pi_{4}$ & $\pi_{5}$ & $\sigma_{1}$ & $\sigma_{2}$ & $\sigma_{3}$ \\
\hline GS & 1.000 & 0.000 & 0.000 & 0.000 & 0.000 & 0.000 & 0.002 & 0.001 & 0.001 \\
$\pi_{1}$ & 0.000 & 1.000 & 0.117 & 0.003 & 0.014 & 0.003 & 0.000 & 0.000 & 0.000 \\
$\pi_{2}$ & 0.000 & 0.117 & 1.000 & 0.019 & 0.043 & 0.002 & 0.000 & 0.000 & 0.000 \\
$\pi_{3}$ & 0.000 & 0.003 & 0.019 & 1.000 & 0.005 & 0.102 & 0.000 & 0.000 & 0.000 \\
$\pi_{4}$ & 0.000 & 0.014 & 0.043 & 0.005 & 1.000 & 0.015 & 0.000 & 0.000 & 0.000 \\
$\pi_{5}$ & 0.000 & 0.003 & 0.002 & 0.102 & 0.015 & 1.000 & 0.000 & 0.000 & 0.000 \\
$\sigma_{1}$ & 0.002 & 0.000 & 0.000 & 0.000 & 0.000 & 0.000 & 1.000 & 0.121 & 0.113 \\
$\sigma_{2}$ & 0.001 & 0.000 & 0.000 & 0.000 & 0.000 & 0.000 & 0.121 & 1.000 & 0.813 \\
$\sigma_{3}$ & 0.001 & 0.000 & 0.000 & 0.000 & 0.000 & 0.000 & 0.113 & 0.813 & 1.000 \\
\hline
\end{tabular}

Table S8: Absolute value of the overlap integral between the PFP ground state and F4 core electron excited Slater determinants.

\begin{tabular}{c|ccccccc}
\hline & GS & $\pi_{1}$ & $\pi_{2}$ & $\pi_{3}$ & $\sigma_{1}$ & $\sigma_{2}$ & $\sigma_{3}$ \\
\hline GS & 1.000 & 0.000 & 0.000 & 0.000 & 0.002 & 0.000 & 0.000 \\
$\pi_{1}$ & 0.000 & 1.000 & 0.076 & 0.016 & 0.000 & 0.000 & 0.000 \\
$\pi_{2}$ & 0.000 & 0.076 & 1.000 & 0.097 & 0.000 & 0.000 & 0.000 \\
$\pi_{3}$ & 0.000 & 0.016 & 0.097 & 1.000 & 0.000 & 0.000 & 0.000 \\
$\sigma_{1}$ & 0.002 & 0.000 & 0.000 & 0.000 & 1.000 & 0.068 & 0.058 \\
$\sigma_{2}$ & 0.000 & 0.000 & 0.000 & 0.000 & 0.068 & 1.000 & 0.767 \\
$\sigma_{3}$ & 0.000 & 0.000 & 0.000 & 0.000 & 0.058 & 0.767 & 1.000 \\
\hline
\end{tabular}




\section{S2.1.5 HMO Calculations}

Molecular orbital scheme for perfluoropentacene as obtained from Hückel molecular orbital calculations. The parameter for the Coulomb integral of each carbon center not connected to a fluorine atom was $\alpha$, the parameter for the reduced resonance integral between directly connected carbon centers was $\beta$. The parameter for the Coulomb integral of each fluorine center was $\alpha+3.0 \beta$, the parameter for the reduced resonance integral between carbon centers directly connected to fluorine was chosen as $0.7 \beta$. To account for inductive effects due to the fluorine substituent, the Coulomb integral at such carbon centers directly connected to fluorine was $\alpha+0.3 \beta$.

Shown is the diagram of orbital energies $\epsilon_{i}$ and the corresponding schemes for the various molecular orbitals $\phi_{i}$, which are expanded as a linear combination of atomic orbitals (LCAO) according to $\phi_{i}=\sum_{\mu} c_{\mu i} \chi_{\mu}$. Atomic orbitals $\chi_{\mu}$ are assumed to be orthonormalized. The specific molecular structure is only shown to visualize the chosen topology of the $\pi$-system. Each vertex corresponds to an atomic p-center and each edge indicates directly connected p-centers of the $\pi$-system. The radius of the circles at the various centers for a given molecular orbital $\phi_{i}$ was chosen proportional to the absolute value of the corresponding LCAO-MO coefficient $c_{\mu i}$ at this center. The relative sign is encoded in filled circles or unfilled circles. The symmetry assignment of molecular orbitals was made assuming $D_{2 h}$ point group symmetry of the equilibrium structure of perfluoropentacene. An orientation according to the Mulliken convention was assumed for designation of irreducible representations: The $z$-axis goes through most atoms, $x$-axis is normal to ring plane. Note that a different orientation was used in the Kohn-Sham DFT calculations reported above. 


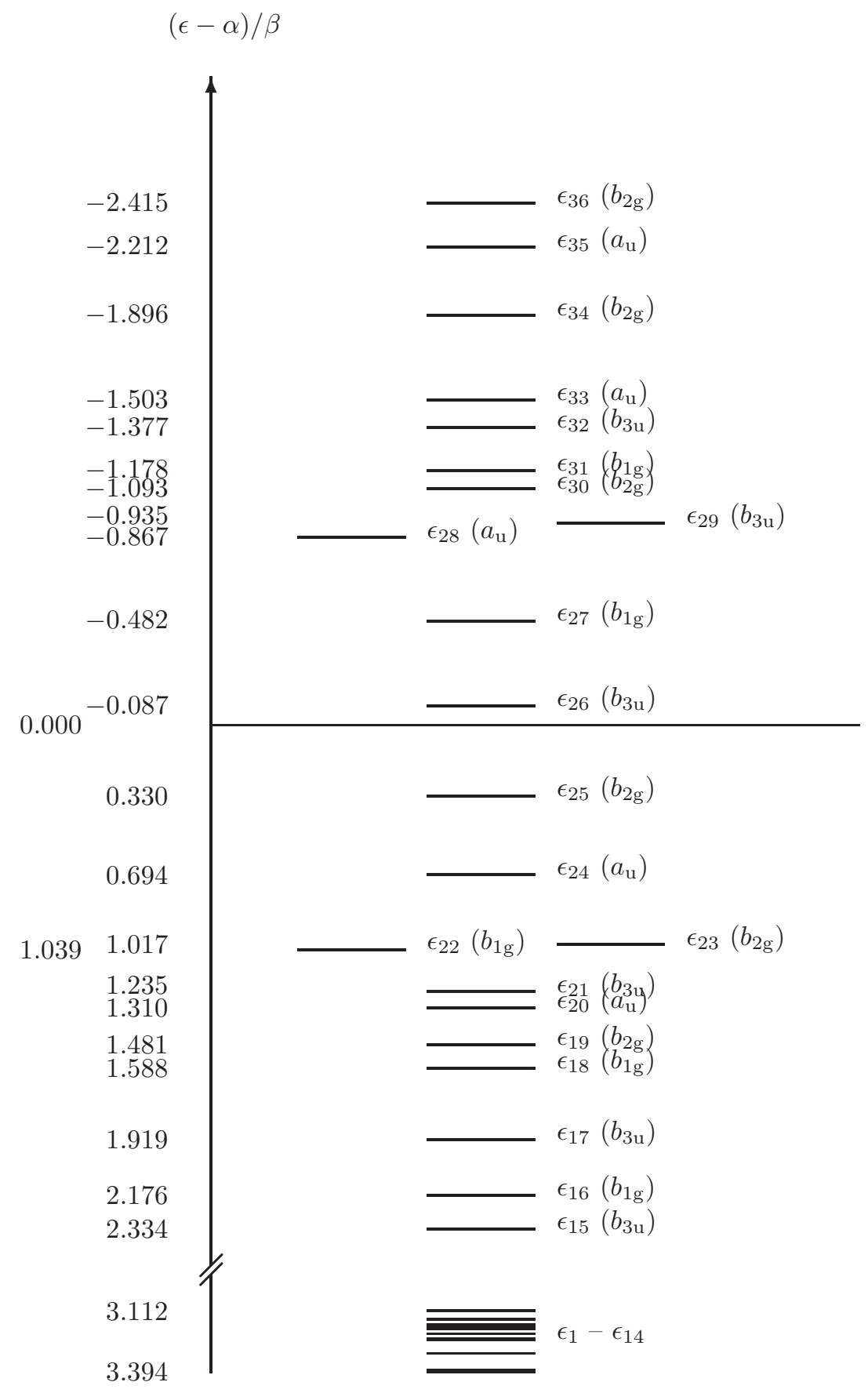



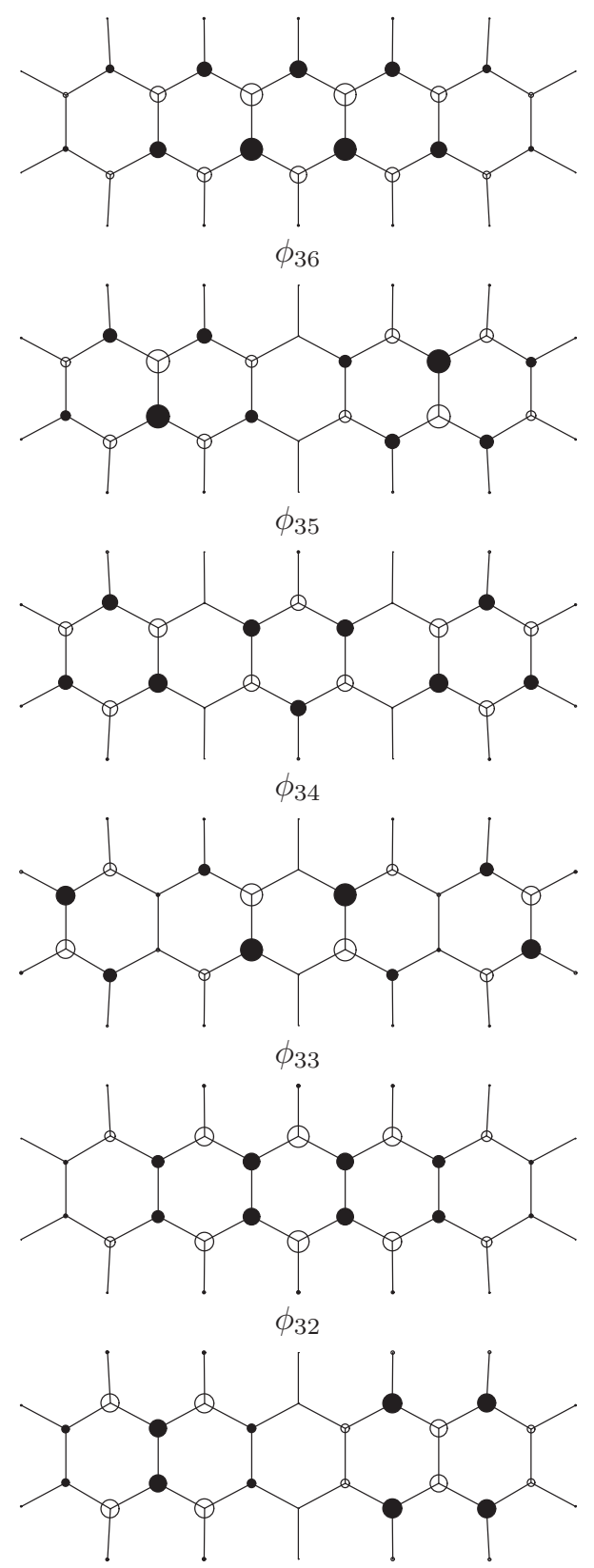

$\phi_{31}$ 

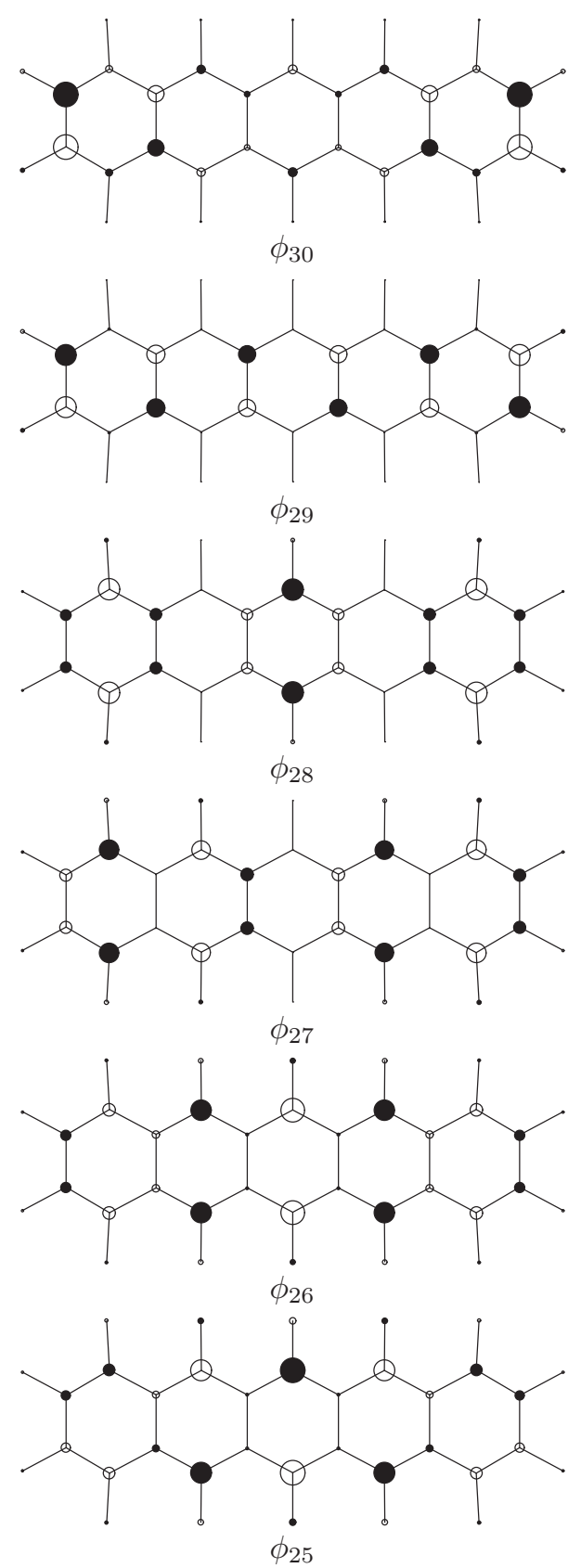

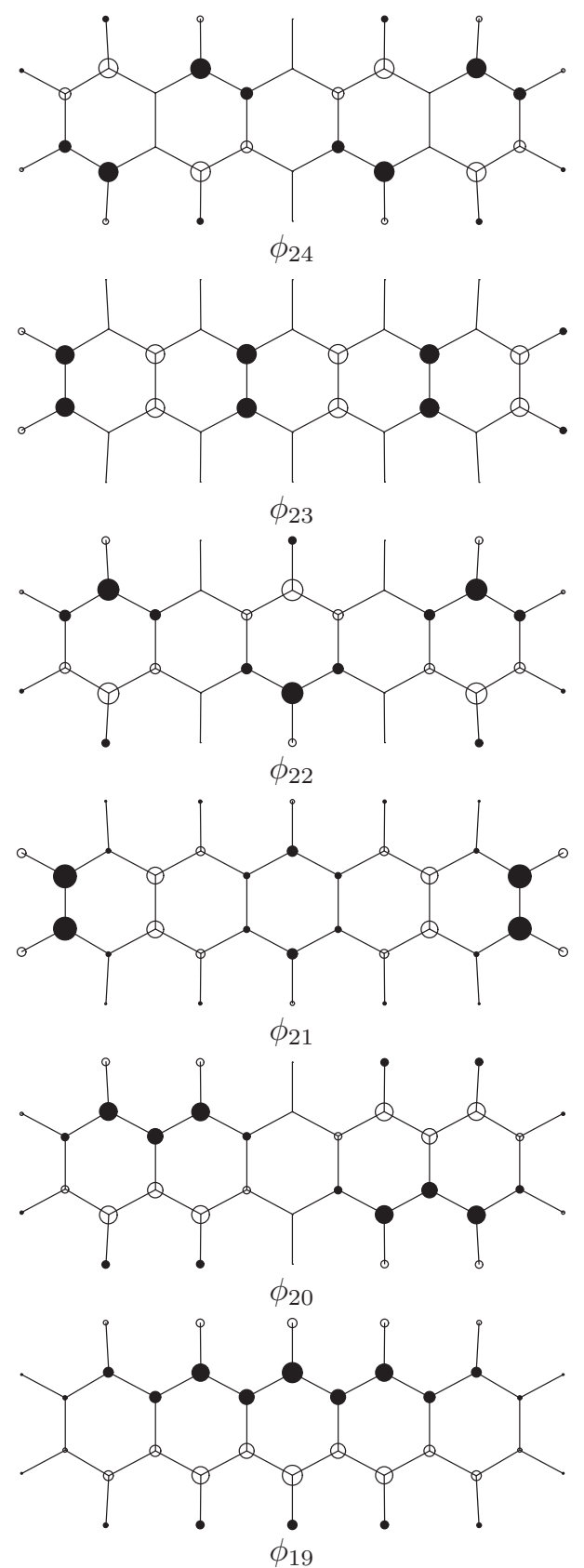

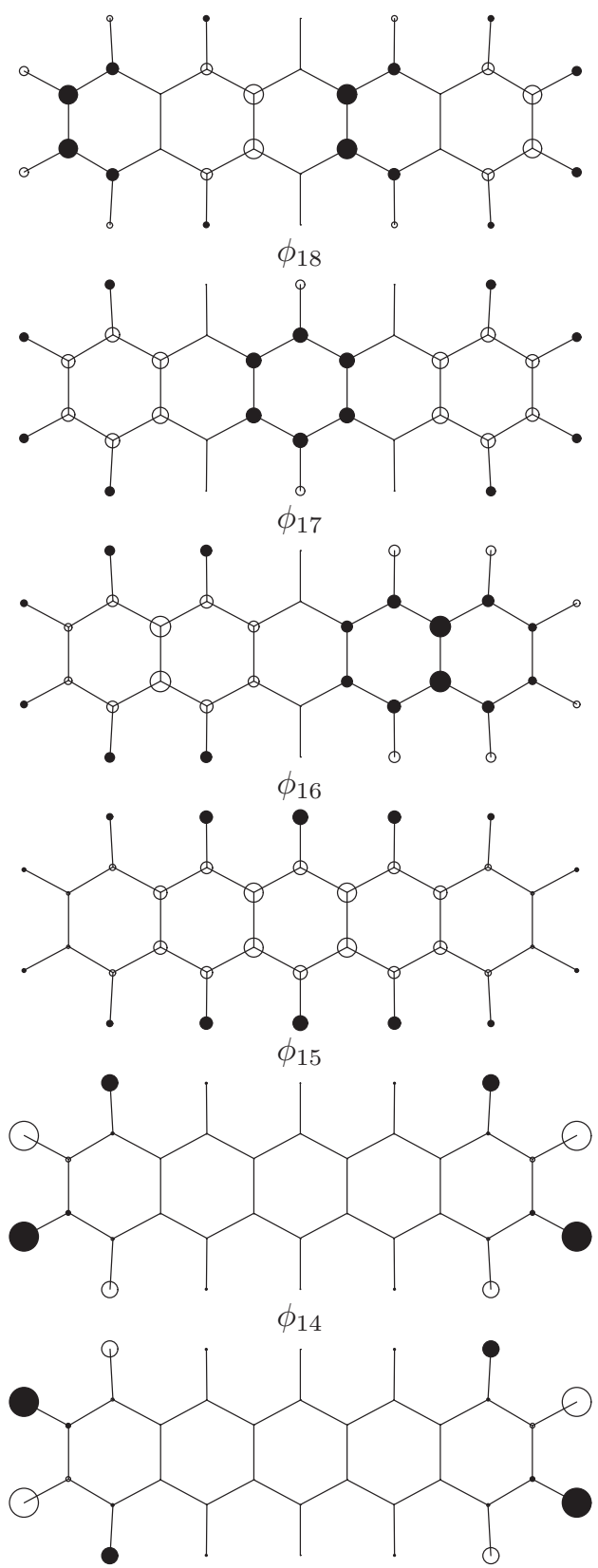

$\phi_{13}$ 

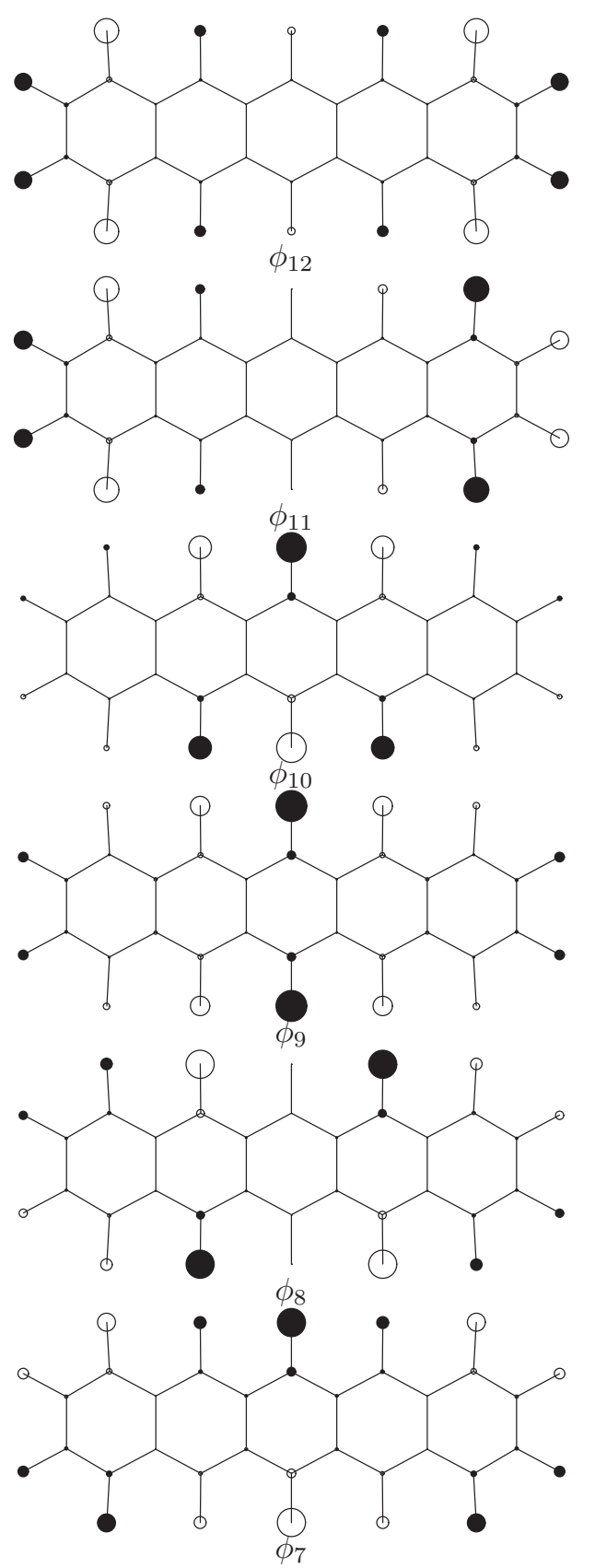

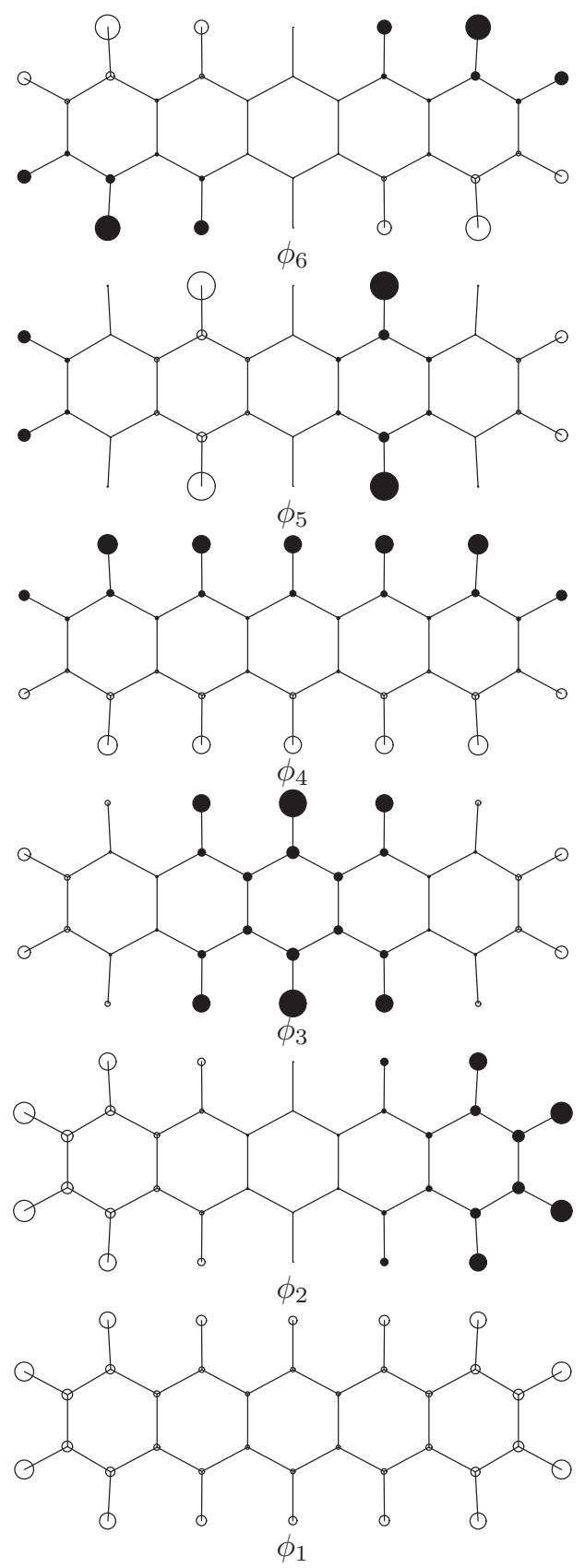
S2.1.6 Generalized MO-diagrams of $\pi$ interactions in PFP and the allyl molecule 


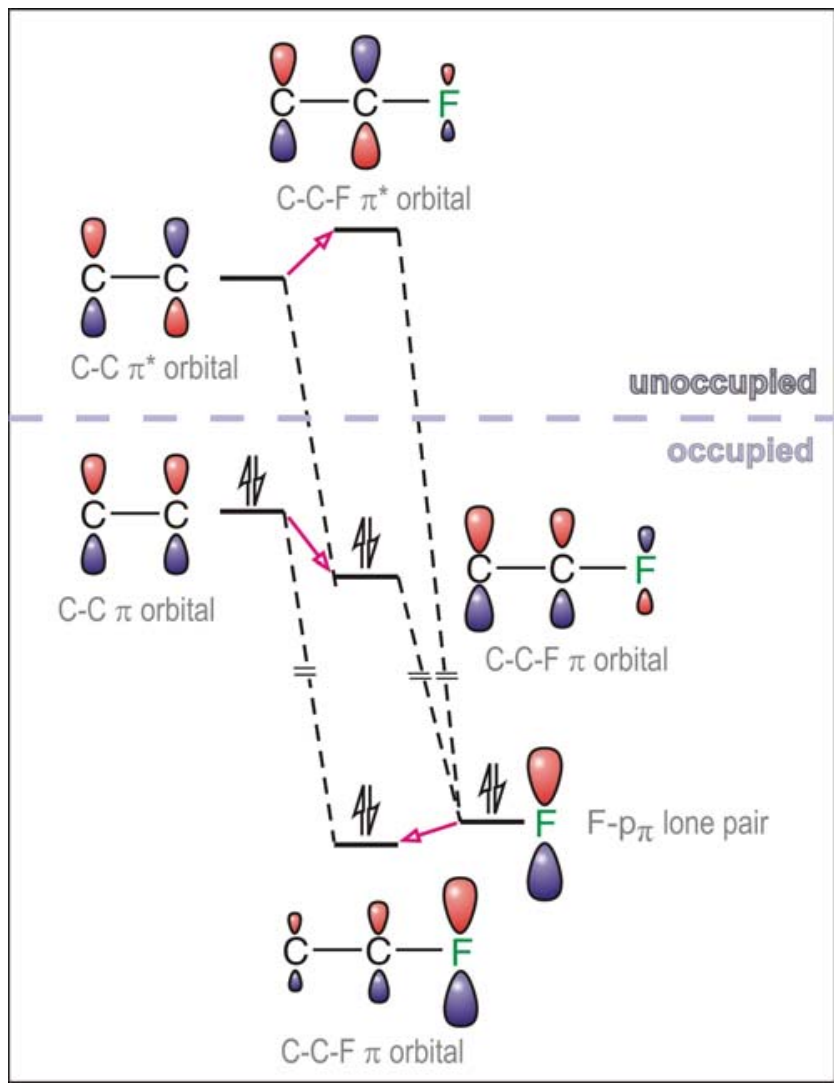

Figure S13: Generalized MO-diagram of the $\pi$-interaction between fluorine and the $\pi$-system of an aromatic carbon backbone. 


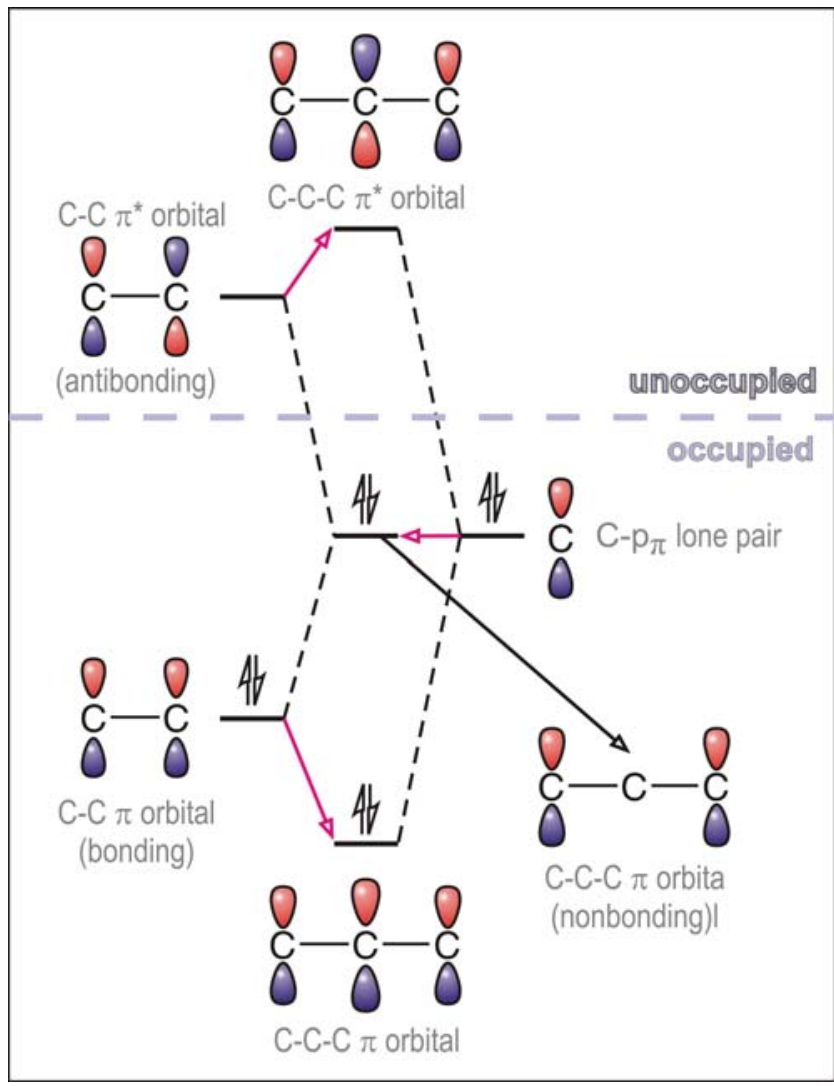

Figure S14: Generalized MO-diagram of the $\pi$-interaction between carbon and the $\pi$-system in an allyl molecule. 


\section{S2.2 Pentacene}

\section{S2.2.1 HMO Calculations}

Molecular orbital scheme for pentacene as obtained from Hückel molecular orbital calculations. The parameter for the Coulomb integral of each carbon center was $\alpha$, the parameter for the reduced resonance integral between directly connected carbon centers was $\beta$.

Shown is the diagram of orbital energies $\epsilon_{i}$ and the corresponding schemes for the various molecular orbitals $\phi_{i}$, which are expanded as a linear combination of atomic orbitals (LCAO) according to $\phi_{i}=\sum_{\mu} c_{\mu i} \chi_{\mu}$. Atomic orbitals $\chi_{\mu}$ are assumed to be orthonormalized. The specific molecular structure is only shown to visualize the chosen topology of the $\pi$-system. Each vertex corresponds to an atomic p-center and each edge indicates directly connected p-centers of the $\pi$-system. The radius of the circles at the various centers for a given molecular orbital $\phi_{i}$ was chosen proportional to the absolute value of the corresponding LCAO-MO coefficient $c_{\mu i}$ at this center. The relative sign is encoded in filled circles or unfilled circles. The symmetry assignment of molecular orbitals was made assuming $\mathrm{D}_{2 \mathrm{~h}}$ point group symmetry of the equilibrium structure of pentacene. An orientation according to the Mulliken convention was assumed for designation of irreducible representations: The $z$-axis goes through most atoms, $x$-axis is normal to ring plane. 


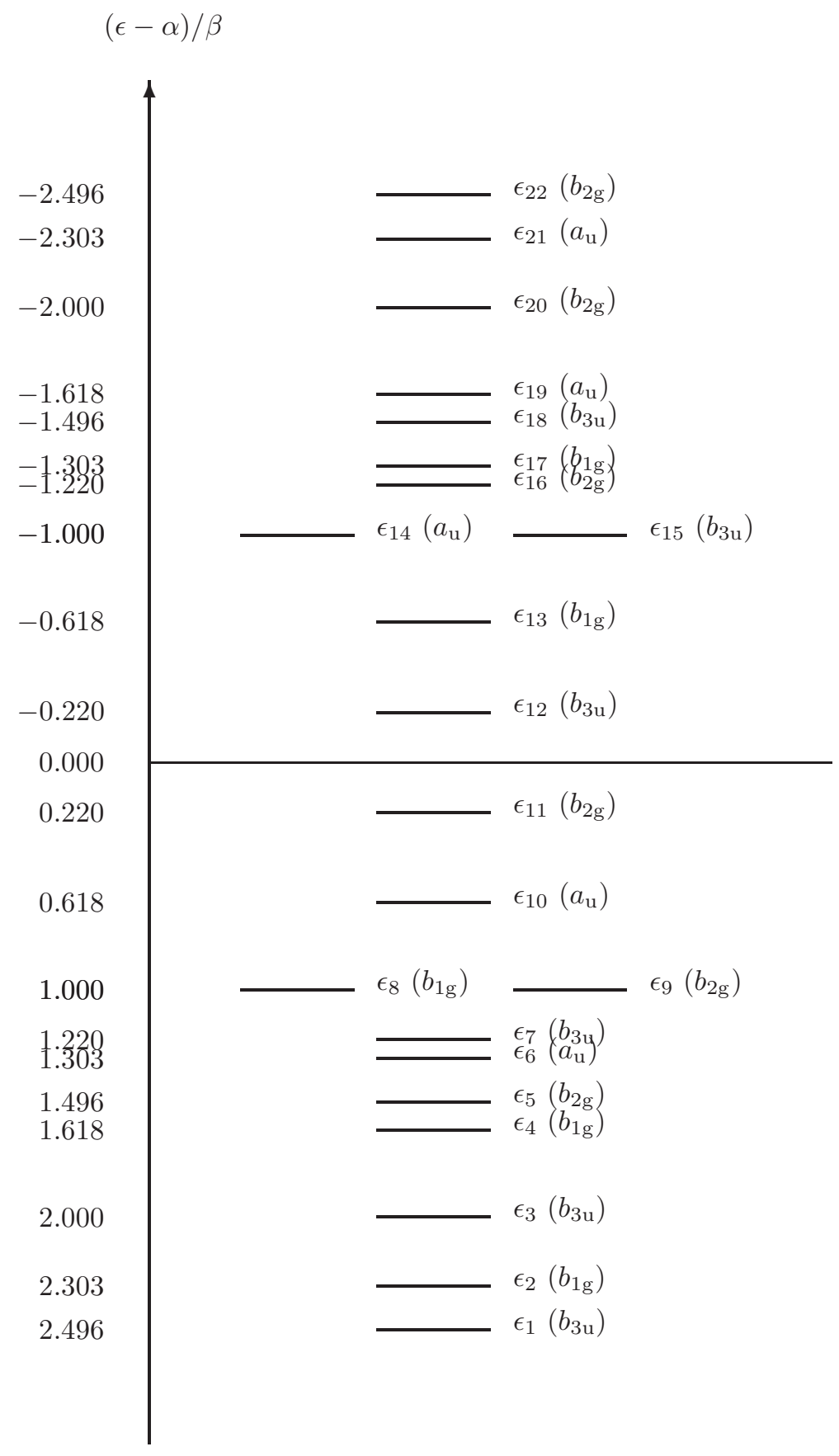



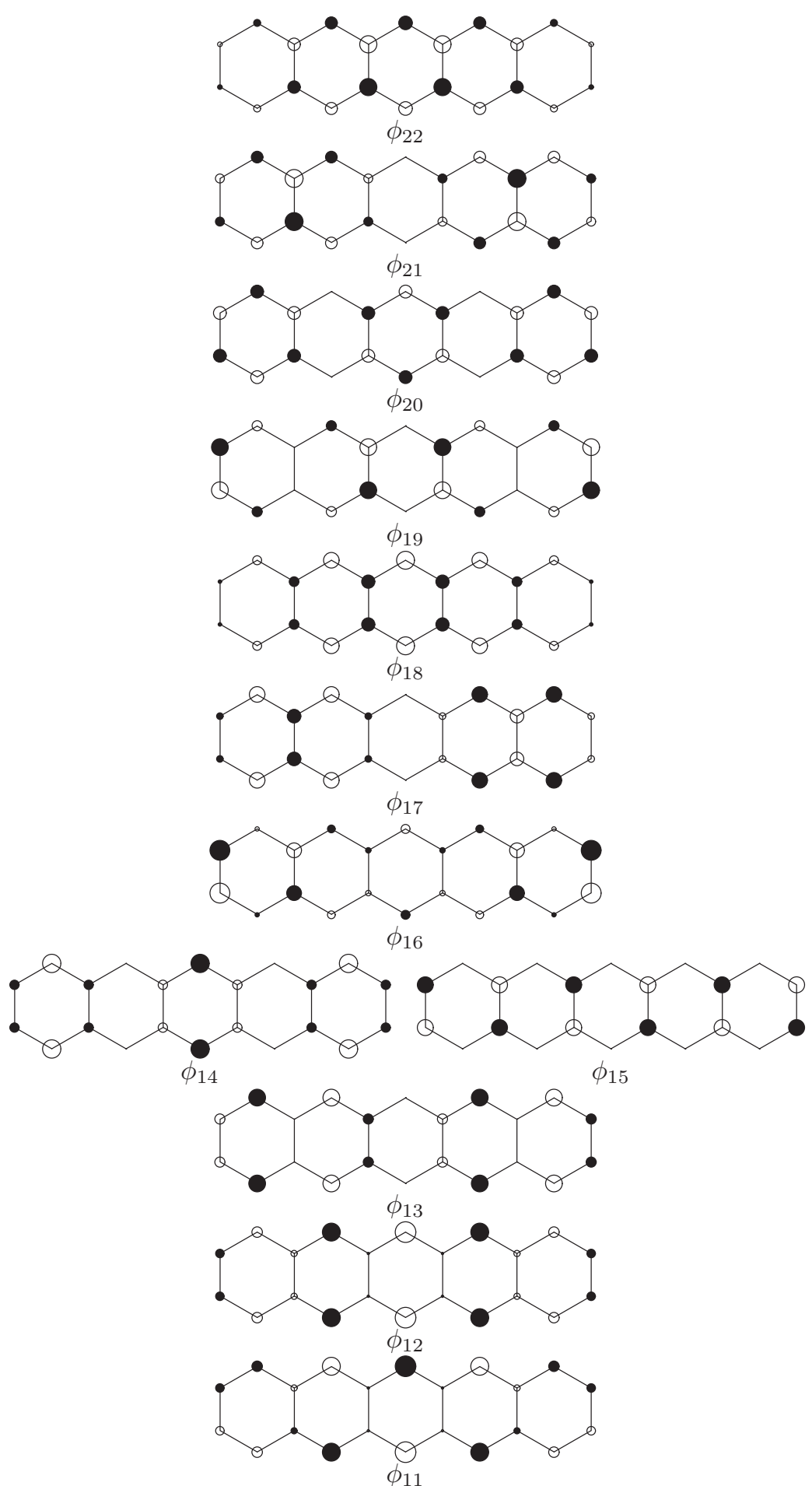


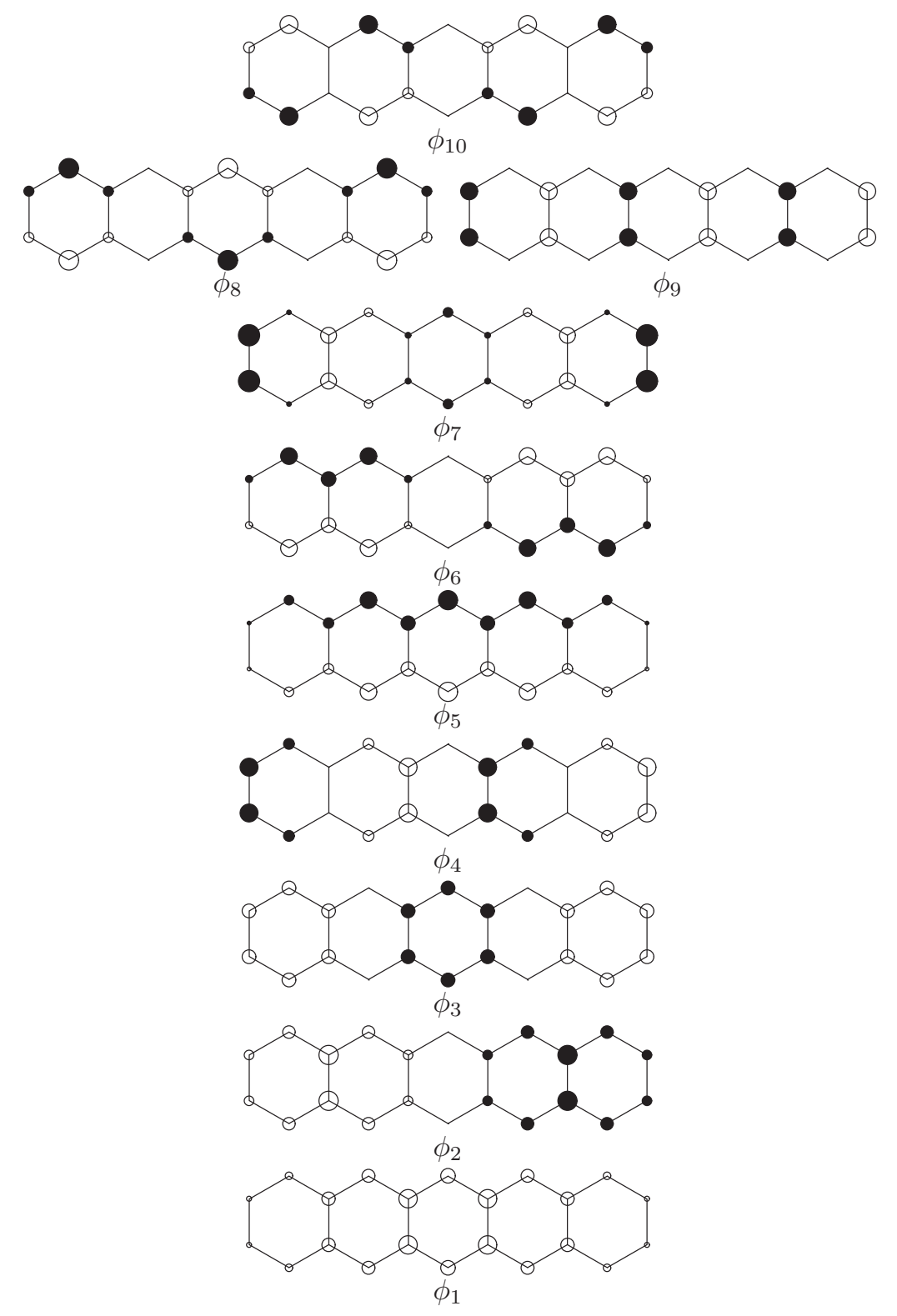




\section{S2.3 Perfluoronaphthalene}

\section{S2.3.1 Molecular orbitals}

The figures in this subsection show select Kohn-Sham molecular orbitals as obtained for perfluoronaphthalene with the def2-SVP basis set and the PBE density functional as implemented in the program package Turbomole 6.6 [23], [25], utilizing the RI approximation [23]-[25]. The Kohn-Sham molecular orbitals in Figs. S15-S17 were obtained in Kohn-Sham DFT calculations for the electronic ground state. The molecular orbitals shown in Figs. S18-S19 are the results from individual self-consistent field (SCF) calculations that essentially describe single electron excitations from localized F1s core orbitals (centered at F1 or F2 which are indicated by the green color, whereas other fluorine atoms are shown in blue color; see also main text for the numbering convention) into different unoccupied molecular orbitals. For each of these SCF calculations only the obtained energetically high-lying molecular orbital, into which the electron is promoted, is shown. This orbital we loosely refer to as a singly occupied molecular orbital (SOMO), as if it were obtained from a restricted open-shell SCF calculation. Note that the actual calculations for excited states were performed in an unrestricted Kohn-Sham framework. The initial Kramers degeneracy of the ground state Kohn-Sham orbitals is thereby lifted such that essentially all occupied molecular orbitals become singly occupied spin-orbitals (different orbitals for different spin, DODS) instead of doubly occupied or singly occupied spatial orbitals. Neither the corresponding relaxed core holes obtained in each of these unrestricted SCF procedures nor the corresponding orbitals with opposite spin are explicitly shown.

The molecular orbitals shown in the first set below are linear combinations of the fluorine core orbitals (Fig. S15). To the second set of molecular orbitals belong energetically low-lying $\pi^{*}$ type Kohn-Sham molecular orbitals (Fig. S16), with LUMO referring to the energetically lowest unoccupied molecular orbital in the electronic groundstate and $\mathrm{LUMO}+1$ referring to the next higher energy molecular orbital. The third set of molecular orbitals contains $\sigma^{*}$ type Kohn-molecular orbitals (Fig. S17). The orbitals of these first three sets were obtained in Kohn-Sham DFT calculations for the electronic ground state, whereas the forth and fifth set (Figs. S18-S19) feature SOMOs from SCF calculations for core-hole excited states. The molecular orbitals are displayed as contour surfaces for a given isovalue $\left(0.05 a_{0}^{-3 / 2}\right)$ of the corresponding oneelectron wavefunction. The different colors (blue $(+1)$ and red $(-1))$ indicate the sign of the isovalue. 

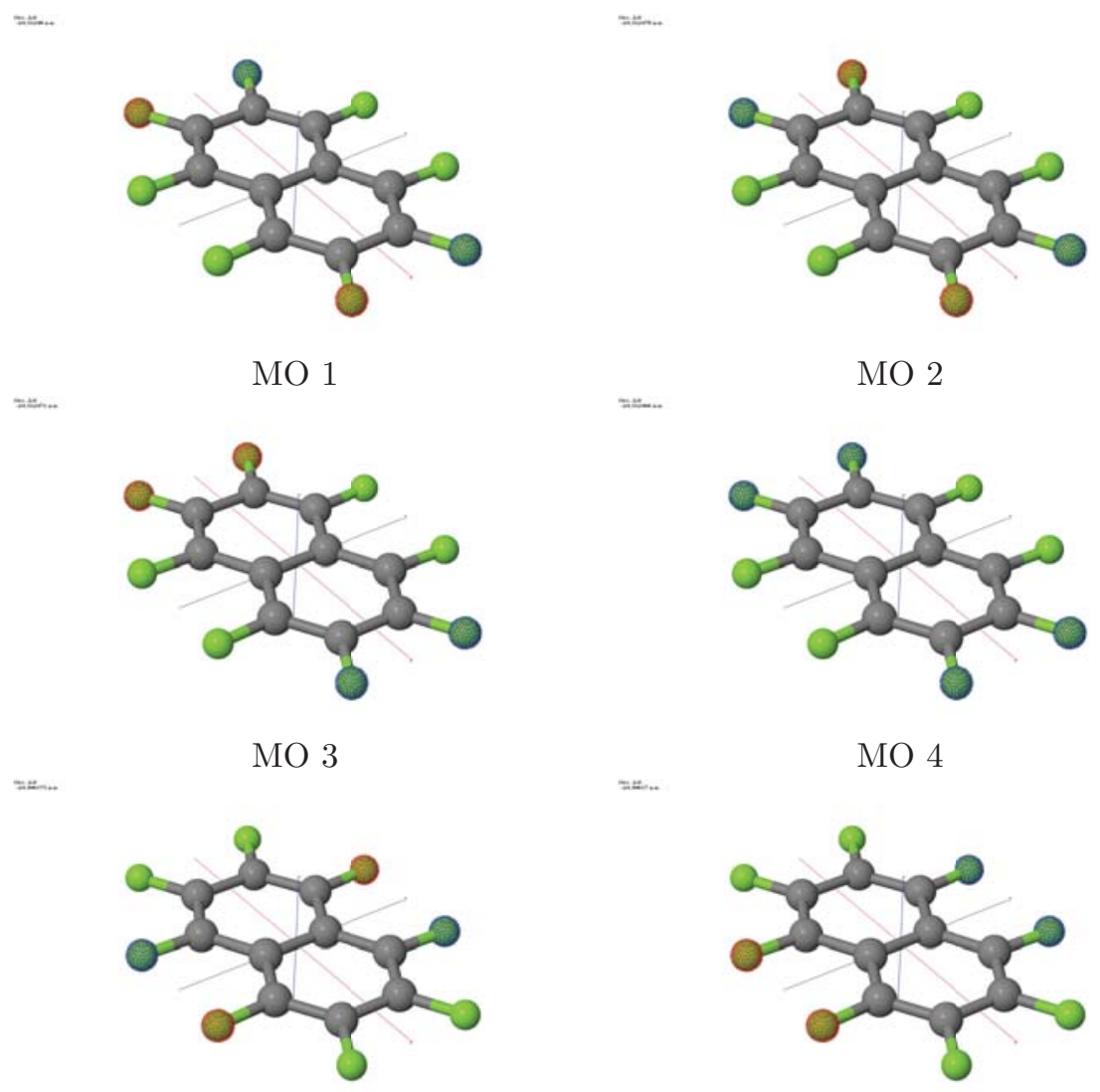

MO 5

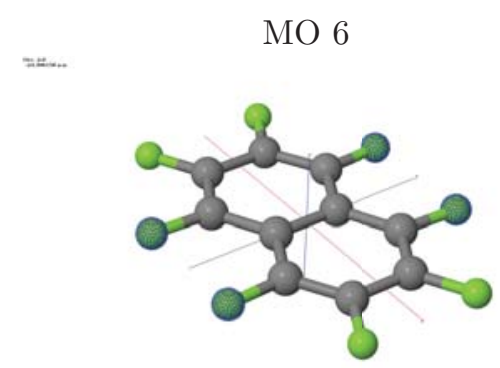

MO 7

MO 8

Figure S15: PFN fluorine core electron MOs. 

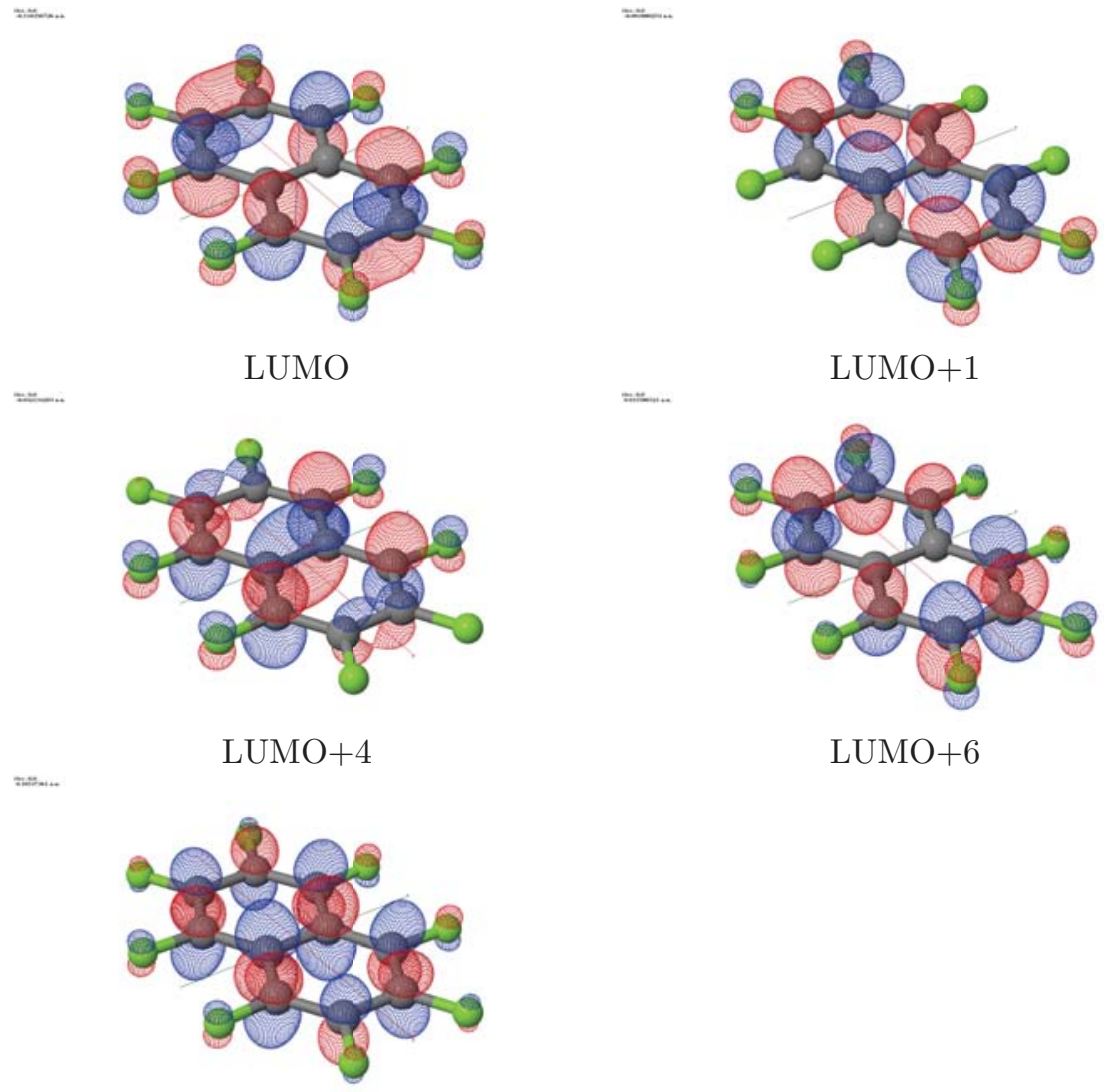

LUMO+6

LUMO +12

Figure S16: PFN $\pi^{*}$ type unoccupied MOs. 

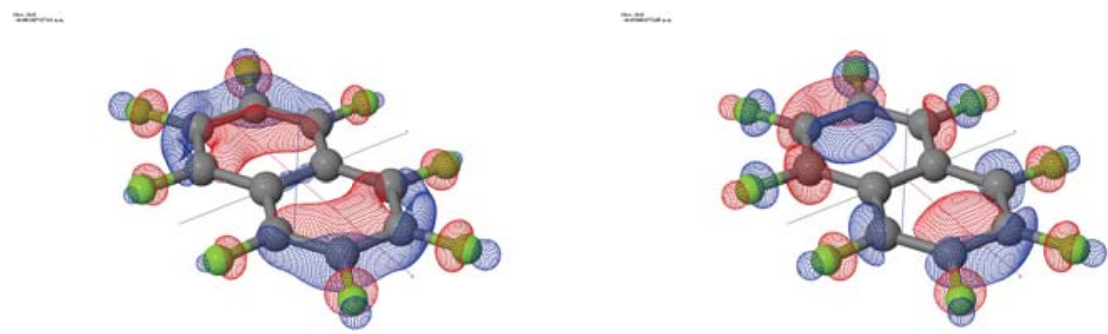

$\mathrm{LUMO}+2$

$\mathrm{LUMO}+3$

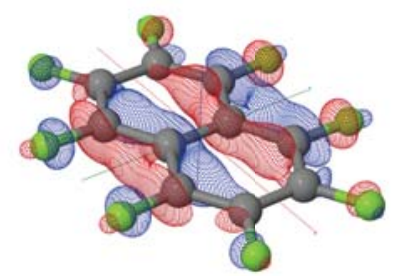

$=$

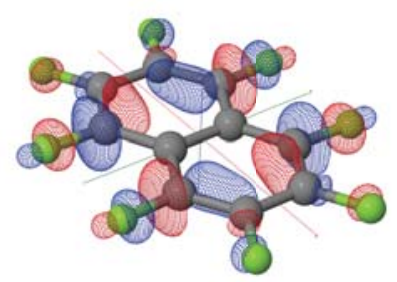

$\mathrm{LUMO}+5$

LUMO +7

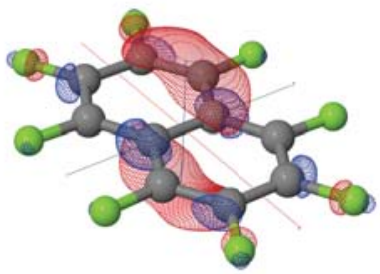

$\mathrm{LUMO}+8$

Figure S17: PFN $\sigma^{*}$ type unoccupied MOs. 

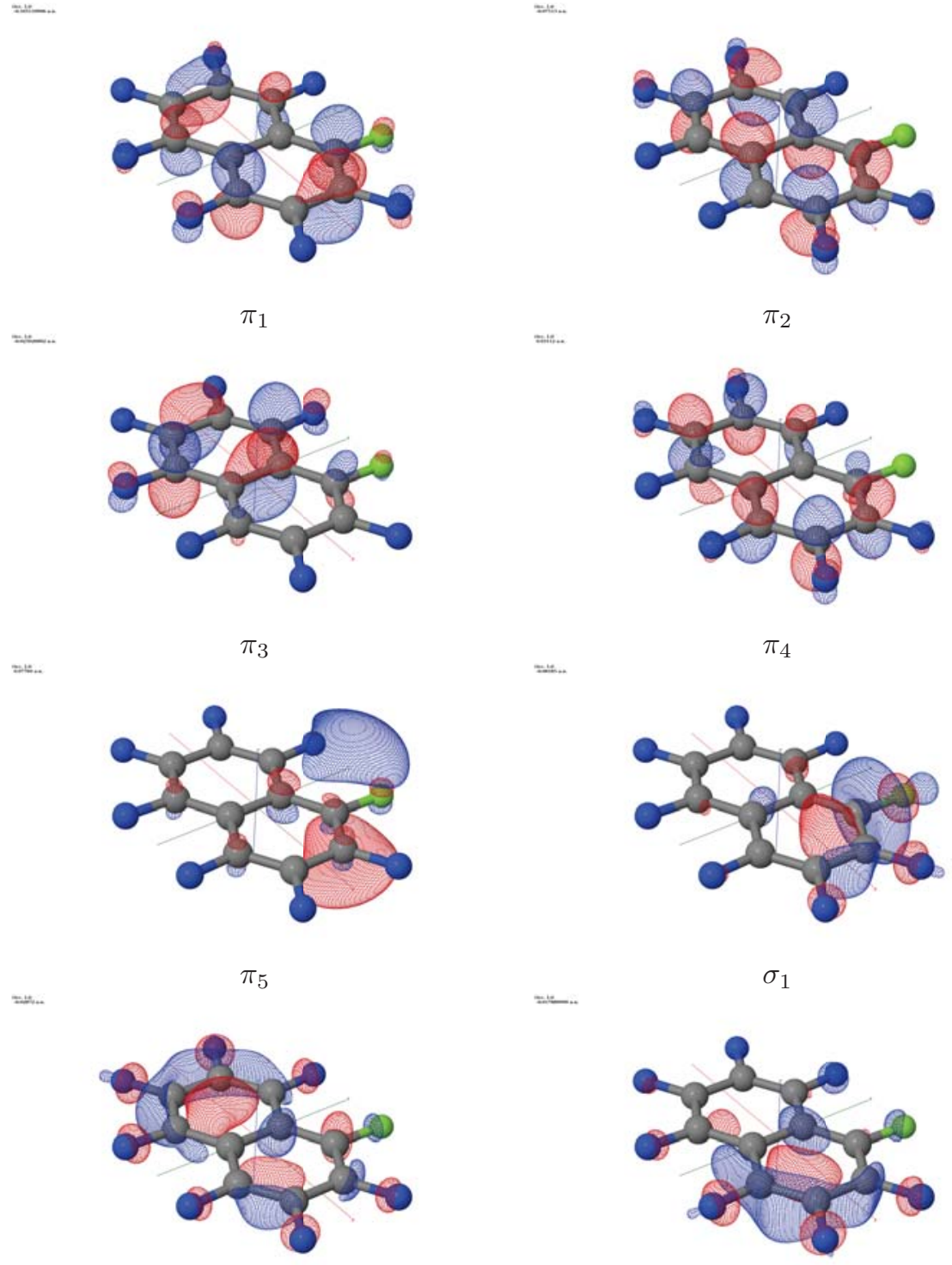

$\sigma_{2}$

$\sigma_{3}$

Figure S18: SOMOs of PFN (resulting from F1 core electron excitations) for the transitions into the lowest-lying MOs. 


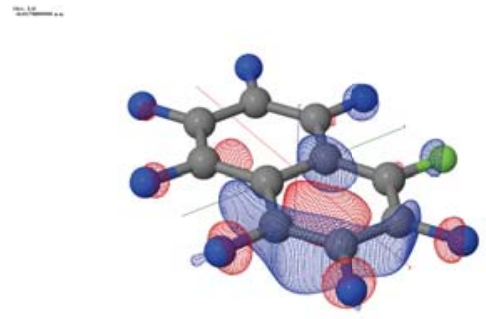

$\sigma_{3}$

min.

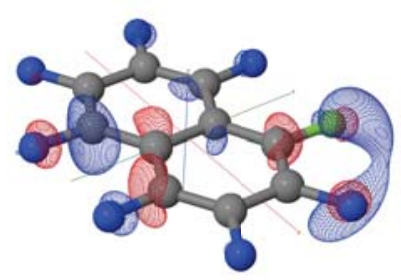

$\sigma_{5}$

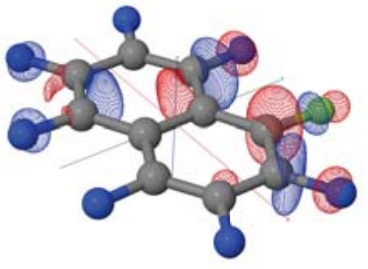

$\sigma_{4}$

Figure S18 (Cont.): SOMOs of PFN (resulting from F1 core electron excitations) for the transitions into the lowest-lying MOs. 


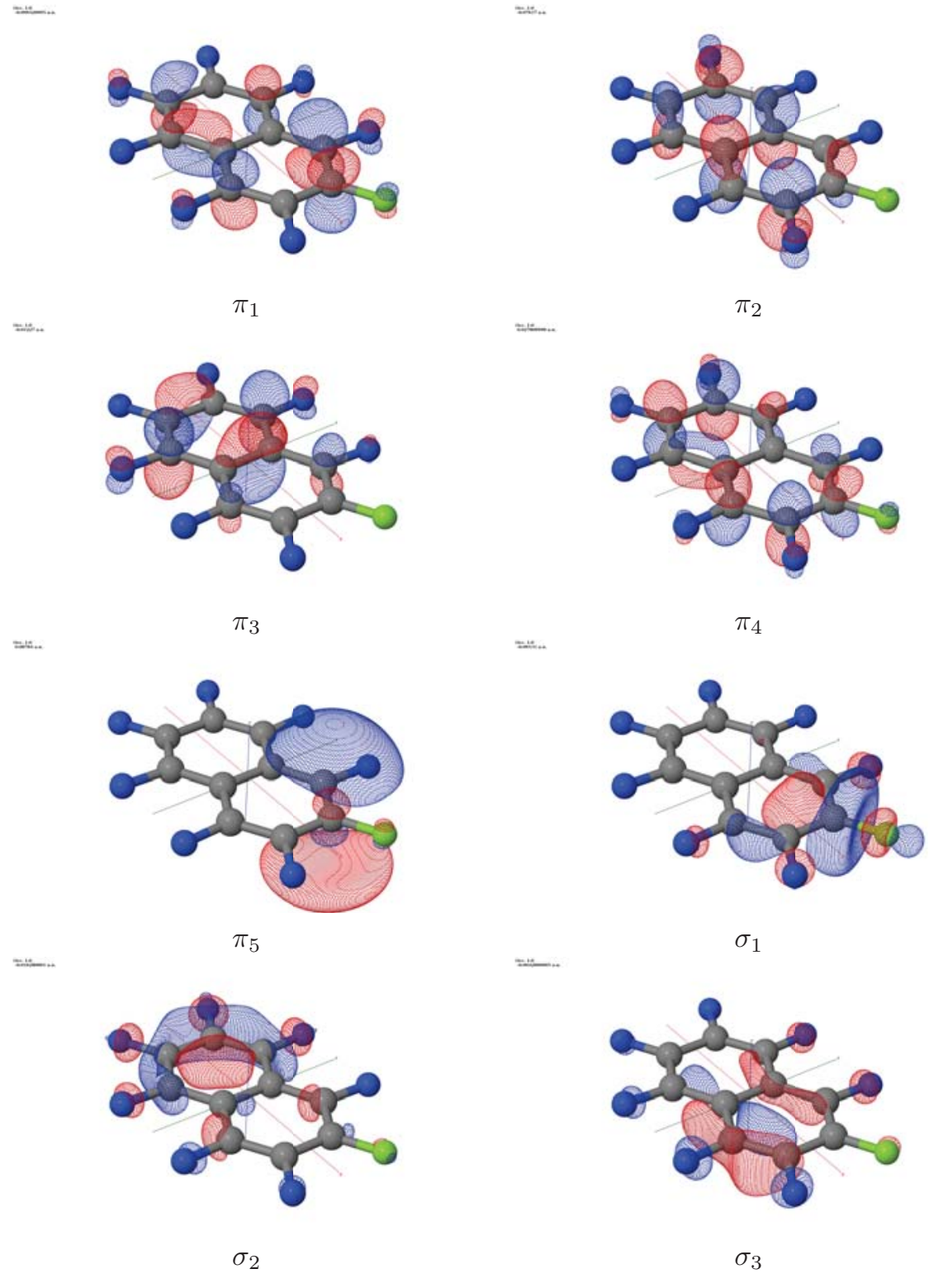

Figure S19: SOMOs of PFN (resulting from F2 core electron excitations) for the transitions into the lowest-lying MOs. 


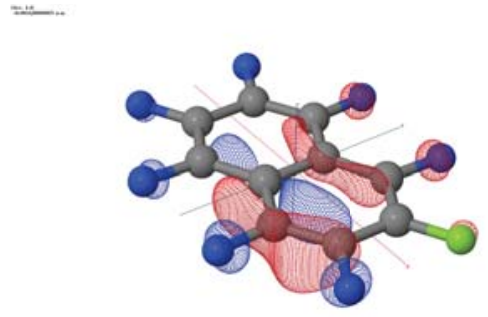

$=$

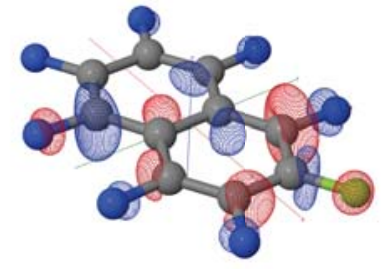

$\sigma_{4}$

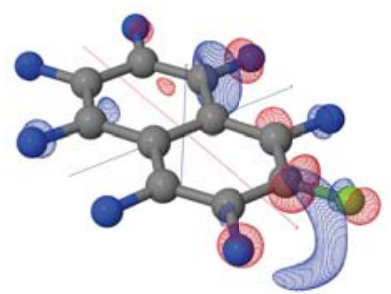

$\sigma_{5}$

Figure S19 (Cont.): SOMOs of PFN (resulting from F2 core electron excitations) for the transitions into the lowest-lying MOs. 
S2.3.2 Calculated excitations, transition dipole moments and oscillator strengths

Table S9: PFN F2 core electron excitation energies, oscillator strengths and transition dipole moment components for the transition into the lowest lying unoccupied MOs. Method: $\triangle \mathrm{SCF}$, revPBE/StoBe basis set (TZ quality).

\begin{tabular}{ccrrrr}
\hline Exc. & $\varepsilon / \mathbf{e V}$ & Osc. Str. & TDM $_{\mathbf{x}}$ & TDM $_{\mathbf{y}}$ & $\mathbf{T D M}_{\mathbf{z}}$ \\
\hline$\pi_{1}$ & 686.987 & 0.002525 & 0.000000 & 0.000000 & -0.012233 \\
$\pi_{2}$ & 687.821 & 0.000051 & 0.000000 & 0.000000 & -0.001729 \\
$\pi_{3}$ & 689.061 & 0.000912 & 0.000000 & 0.000000 & -0.007349 \\
$\pi_{4}$ & 690.709 & 0.000237 & 0.000000 & 0.000000 & -0.003697 \\
$\pi_{5}$ & 692.699 & 0.001867 & 0.000000 & 0.000000 & 0.010465 \\
$\sigma_{1}$ & 687.199 & 0.010321 & -0.000420 & -0.024755 & 0.000000 \\
$\sigma_{2}$ & 689.308 & 0.000574 & -0.002407 & -0.005285 & 0.000000 \\
$\sigma_{3}$ & 689.619 & 0.000541 & 0.005010 & 0.002537 & 0.000000 \\
$\sigma_{4}$ & 690.877 & 0.002962 & 0.001752 & 0.013101 & 0.000000 \\
$\sigma_{5}$ & 691.450 & 0.002151 & -0.007681 & -0.008225 & 0.000000 \\
\hline
\end{tabular}

Table S10: PFN F3 core electron excitation energies, oscillator strengths and transition dipole moment components for the transition into the lowest lying unoccupied MOs. Method: $\triangle \mathrm{SCF}$, revPBE/StoBe basis set (TZ quality).

\begin{tabular}{ccrrrr}
\hline Exc. & $\varepsilon / \mathbf{~ e V}$ & Osc. Str. & TDM $_{\mathbf{x}}$ & \multicolumn{1}{c}{$\mathbf{T D M}_{\mathbf{y}}$} & \multicolumn{1}{c}{$\mathbf{T D M}_{\mathbf{z}}$} \\
\hline$\pi_{1}$ & 687.337 & 0.002560 & 0.000000 & 0.000000 & -0.012345 \\
$\pi_{2}$ & 687.983 & 0.000775 & 0.000000 & 0.000000 & 0.006776 \\
$\pi_{3}$ & 689.641 & 0.000084 & 0.000000 & 0.000000 & 0.002304 \\
$\pi_{4}$ & 690.829 & 0.000982 & 0.000000 & 0.000000 & -0.007597 \\
$\pi_{5}$ & 693.110 & 0.000934 & 0.000000 & 0.000000 & -0.007433 \\
$\sigma_{1}$ & 687.108 & 0.010572 & -0.021869 & -0.012225 & 0.000000 \\
$\sigma_{2}$ & 689.861 & 0.000524 & 0.005401 & -0.001317 & 0.000000 \\
$\sigma_{3}$ & 690.233 & 0.000812 & 0.003863 & -0.005778 & 0.000000 \\
$\sigma_{4}$ & 691.367 & 0.000034 & 0.001098 & -0.000599 & 0.000000 \\
$\sigma_{5}$ & 691.726 & 0.003186 & 0.013527 & 0.002243 & 0.000000 \\
\hline
\end{tabular}


S2.3.3 Overlap integrals between ground state and excited states Slater determinants

Table S11: Absolute value of the overlap integral between the PFN ground state and F2 core electron excited Slater determinants.

\begin{tabular}{l|ccccccccccc}
\hline & GS & $\pi_{1}$ & $\pi_{2}$ & $\pi_{3}$ & $\pi_{4}$ & $\pi_{5}$ & $\sigma_{1}$ & $\sigma_{2}$ & $\sigma_{3}$ & $\sigma_{4}$ & $\sigma_{5}$ \\
\hline GS & 1.000 & 0.000 & 0.000 & 0.000 & 0.000 & 0.000 & 0.002 & 0.000 & 0.001 & 0.000 & 0.000 \\
$\pi_{1}$ & 0.000 & 1.000 & 0.022 & 0.138 & 0.010 & 0.003 & 0.000 & 0.000 & 0.000 & 0.000 & 0.000 \\
$\pi_{2}$ & 0.000 & 0.022 & 1.000 & 0.020 & 0.014 & 0.004 & 0.000 & 0.000 & 0.000 & 0.000 & 0.000 \\
$\pi_{3}$ & 0.000 & 0.138 & 0.020 & 1.000 & 0.010 & 0.010 & 0.000 & 0.000 & 0.000 & 0.000 & 0.000 \\
$\pi_{4}$ & 0.000 & 0.010 & 0.014 & 0.010 & 1.000 & 0.001 & 0.000 & 0.000 & 0.000 & 0.000 & 0.000 \\
$\pi_{5}$ & 0.000 & 0.003 & 0.004 & 0.010 & 0.001 & 1.000 & 0.000 & 0.000 & 0.000 & 0.000 & 0.000 \\
$\sigma_{1}$ & 0.002 & 0.000 & 0.000 & 0.000 & 0.000 & 0.000 & 1.000 & 0.043 & 0.117 & 0.071 & 0.002 \\
$\sigma_{2}$ & 0.000 & 0.000 & 0.000 & 0.000 & 0.000 & 0.000 & 0.043 & 1.000 & 0.503 & 0.057 & 0.009 \\
$\sigma_{3}$ & 0.001 & 0.000 & 0.000 & 0.000 & 0.000 & 0.000 & 0.117 & 0.503 & 1.000 & 0.127 & 0.078 \\
$\sigma_{4}$ & 0.000 & 0.000 & 0.000 & 0.000 & 0.000 & 0.000 & 0.071 & 0.057 & 0.127 & 1.000 & 0.078 \\
$\sigma_{5}$ & 0.000 & 0.000 & 0.000 & 0.000 & 0.000 & 0.000 & 0.002 & 0.009 & 0.078 & 0.078 & 1.000 \\
\hline
\end{tabular}

Table S12: Absolute value of the overlap integral between the PFN ground state and F3 core electron excited Slater determinants.

\begin{tabular}{c|ccccccccccc}
\hline & GS & $\pi_{1}$ & $\pi_{2}$ & $\pi_{3}$ & $\pi_{4}$ & $\pi_{5}$ & $\sigma_{1}$ & $\sigma_{2}$ & $\sigma_{3}$ & $\sigma_{4}$ & $\sigma_{5}$ \\
\hline GS & 1.000 & 0.000 & 0.000 & 0.000 & 0.000 & 0.000 & 0.002 & 0.000 & 0.000 & 0.001 & 0.000 \\
$\pi_{1}$ & 0.000 & 1.000 & 0.025 & 0.097 & 0.025 & 0.003 & 0.000 & 0.000 & 0.000 & 0.000 & 0.000 \\
$\pi_{2}$ & 0.000 & 0.025 & 1.000 & 0.039 & 0.010 & 0.006 & 0.000 & 0.000 & 0.000 & 0.000 & 0.000 \\
$\pi_{3}$ & 0.000 & 0.097 & 0.039 & 1.000 & 0.020 & 0.007 & 0.000 & 0.000 & 0.000 & 0.000 & 0.000 \\
$\pi_{4}$ & 0.000 & 0.025 & 0.010 & 0.020 & 1.000 & 0.004 & 0.000 & 0.000 & 0.000 & 0.000 & 0.000 \\
$\pi_{5}$ & 0.000 & 0.003 & 0.006 & 0.007 & 0.004 & 1.000 & 0.000 & 0.000 & 0.000 & 0.000 & 0.000 \\
$\sigma_{1}$ & 0.002 & 0.000 & 0.000 & 0.000 & 0.000 & 0.000 & 1.000 & 0.002 & 0.064 & 0.053 & 0.052 \\
$\sigma_{2}$ & 0.000 & 0.000 & 0.000 & 0.000 & 0.000 & 0.000 & 0.002 & 1.000 & 0.348 & 0.056 & 0.097 \\
$\sigma_{3}$ & 0.000 & 0.000 & 0.000 & 0.000 & 0.000 & 0.000 & 0.064 & 0.348 & 1.000 & 0.094 & 0.024 \\
$\sigma_{4}$ & 0.001 & 0.000 & 0.000 & 0.000 & 0.000 & 0.000 & 0.053 & 0.056 & 0.094 & 1.000 & 0.112 \\
$\sigma_{5}$ & 0.000 & 0.000 & 0.000 & 0.000 & 0.000 & 0.000 & 0.052 & 0.097 & 0.024 & 0.112 & 1.000 \\
\hline
\end{tabular}




\section{S3 References}

[1] Marks, M.; Schmidt, C.; Schwalb, C. H.; Breuer, T.; Witte, G.; Höfer, U. Temperature Dependent Structural Phase Transition at the Perfluoropentacene/Ag(111) Interface. J. Phys. Chem. C 2012, 116, 1904.

[2] Yokoyama, T.; Seki, K.; Morisada, I.; Edamatsu, K.; Ohta, T. X-ray Absorption Spectra of Poly-p-Phenylenes and Polyacenes: Localization of $\pi$ Orbitals. Phys. Scr. 1990, 41, 189.

[3] Stöhr, J. NEXAFS Spectroscopy; Gomer, R. Ed.; Springer: Berlin, Germany, 1992.

[4] Bouchoms, I.; Schoonveld, W.; Vrijmoeth, J.; Klapwijk, T. Morphology Identification of the Thin Film Phases of Vacuum Evaporated Pentacene on $\mathrm{SiO}_{2}$ Substrates. Synth. Met. 1999, 104, 175.

[5] Kiyomura, T.; Nemoto, T.; Yoshida, K.; Minari, T.; Kurata, H.; Isoda, S. Epitaxial Growth of Pentacene Thin-Film Phase on Alkali Halides. Thin Solid Films, 2006, 515, 810 .

[6] Götzen, J.; Käfer, D.; Wöll, C.; Witte, G. Growth and Structure of Pentacene Films on Graphite: Weak Adhesion as a Key for Epitaxial Film Growth. Phys. Rev. B 2010, 81, 085440.

[7] Kowarik, S.; Gerlach, A.; Hinderhofer, A.; Milita, S.; Borgatti, F.; Zontone, F.; Suzuki, T.; Biscarini, F.; Schreiber, F. Structure, Morphology, and Growth Dynamics of Perfluoro-pentacene Thin Films. Phys. Status Solidi RRL 2008, 2, 120 .

[8] Breuer, T.; Witte, G. Epitaxial Growth of Perfluoropentacene Films with Predefined Molecular Orientation: A Route for Single-Crystal Optical Studies. Phys. Rev. B 2011, 83, 155428.

[9] Breuer, T.; Salzmann, I.; Götzen, J.; Oehzelt, M.; Morherr, A.; Koch, N.; Witte, G. Interrelation between Substrate Roughness and Thin-Film Structure of Functionalized Acenes on Graphite. Cryst. Growth Des. 2011, 11, 4996.

[10] Salzmann, I.; Moser, A.; Oehzelt, M.; Breuer, T.; Feng, X.; Juang, Z.-Y.; Nabok, D.; Della Valle, R. D.; Duhm, S.; Heimel, G.; et al. Epitaxial Growth of $\pi$-Stacked Perfluoropentacene on Graphene-Coated Quartz. ACS Nano 2012, 
6,10874 .

[11] Vinogradov, A.S.; Fedoseenko, S.I.; Krasnikov, S.A.; Preobrajenski, A.B.; Sivkov, V.N.; Vyalikh, D.V.; Molodtsov, S.L.; Adamchuk, V.K.; Laubschat, C.; Kaindl, G. Low-lying Unoccupied Electronic States in 3d Transition-metal Fluorides Probed by NEXAFS at the F1s Threshold. Phys. Rev. B 2005, 71, 045127 .

[12] Vinogradov, A.S.; Dukhnyakov, A.Y.; Ipatov, V.M.; Onopko, D.E.; Pavlychev, A.A.; Titov, S.A. Fine Structure of X-ray Absorption Spectra of the $\left(\mathrm{TiF}_{6}\right)^{2-}$ Radical. Sov. Phys. Solid State 1982, 24, 803-806.

[13] Beyer, P.; Breuer, T.; Ndiaye, S.; Zykov, A.; Viertel, A.; Gensler, M.; Rabe, J. P.; Hecht, S.; Witte, G.; Kowarik, S. Lattice Matching as the Determining Factor for Molecular Tilt and Multilayer Growth Mode of the Nanographene Hexa-peri-hexabenzocoronene. ACS Appl. Mater. Interfaces 2014, 6, 21484.

[14] Duhm S.; Hosoumi, S.; Salzmann, I.; Gerlach, A.; Oehzelt, M.; Wedl, B.; Lee, T.-L.; Schreiber, F.; Koch, N.; Ueno, N.; Kera, S. Influence of Intramolecular Polar Bonds on Interface Energetics in Perfluoro-pentacene on $\mathrm{Ag}(111)$. Phys. Rev. B 2010, 81, 045418.

[15] Käfer, D.; Witte, G. Evolution of Pentacene Films on $\mathrm{Ag}(111)$ : Growth beyond the First Monolayer. Chem. Phys. Lett. 2007, 442, 376.

[16] Schiefer, S.; Huth, M.; Dobrinevski, A.; Nickel, B. Determination of the Crystal Structure of Substrate-Induced Pentacene Polymorphs in Fiber Structured Thin Films. J. Am. Chem. Soc. 2007, 129, 10316.

[17] Sakamoto, Y.; Suzuki, T.; Kobayashi, M.; Gao, Y.; Fukai, Y.; Inoue, Y.; Sato, F.; Tokito, S. Perfluoropentacene: High-Performance p-n Junctions and Complementary Circuits with Pentacene. J. Am. Chem. Soc. 2004, 126, 8138.

[18] Lee, W. H.; Park, J.; Sim, S. H.; Lim, S.; Kim, K. S.; Hong, B. H., Cho, K. Surface-Directed Molecular Assembly of Pentacene on Monolayer Graphene for High-Performance Organic Transistors. J. Am. Chem. Soc. 2011, 133, 4447.

[19] Siegrist, T.; Kloc, C., Schön, J. H.; Batlogg, B.; Haddon, R. C.; Berg, S.; Thomas, G. A. Enhanced Physical Properties in a Pentacene Polymorph. Angew. Chem. Int. Ed. 2001, 40, 1732. 
[20] Akhmed N.A. Crystal Structure of Octafluoronaphthalene. J. Struct. Chem. 1973, 14, 573.

[21] Ahlrichs, R.; Baer, M.; Haeser, M.; Horn, H.; Koelmel, C. Electronic Structure Calculations on Workstation Computers: The Program System Turbomole. Chem. Phys. Lett. 1989, 162, 165.

[22] Treutler, O.; Ahlrichs, R. Efficient Molecular Numerical Integration Schemes. J. Chem. Phys. 1995, 102, 346.

[23] Eichkorn, K.; Treutler, O.; Oehm, H.; Haeser, M.; Ahlrichs, R.; Auxiliary Basis Sets to Approximate Coulomb Potentials. Chem. Phys. Lett. 1995, 240, 283.

[24] Eichkorn, K.; Weigend, F.; Treutler, O.; Ahlrichs, R. Auxiliary Basis Sets for Main Row Atoms and Transition Metals and their use to Approximate Coulomb Potentials. Theo. Chem. Acc. 1997, 97, 119.

[25] Weigend, F.; Accurate Coulomb-fitting Basis Sets for $\mathrm{H}$ to Rn. Phys. Chem. Chem. Phys. 2006, 8, 1057. 